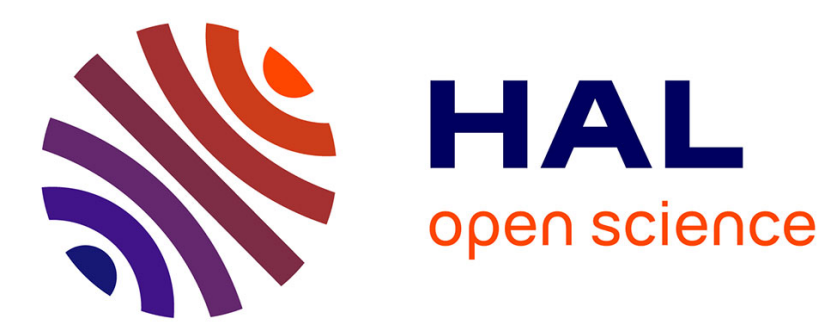

\title{
Synergistic Effect of the TiCl4/p-TsOH Promoter System on the Aza-Prins Cyclization
}

Vianney Durel, Claudia Lalli, Thierry Roisnel, Pierre van de Weghe

\section{To cite this version:}

Vianney Durel, Claudia Lalli, Thierry Roisnel, Pierre van de Weghe. Synergistic Effect of the TiCl4/pTsOH Promoter System on the Aza-Prins Cyclization. Journal of Organic Chemistry, 2016, 81 (3), pp.849-859. 10.1021/acs.joc.5b02342 . hal-01256843

\section{HAL Id: hal-01256843 \\ https://hal-univ-rennes1.archives-ouvertes.fr/hal-01256843}

Submitted on 25 Nov 2020

HAL is a multi-disciplinary open access archive for the deposit and dissemination of scientific research documents, whether they are published or not. The documents may come from teaching and research institutions in France or abroad, or from public or private research centers.
L'archive ouverte pluridisciplinaire HAL, est destinée au dépôt et à la diffusion de documents scientifiques de niveau recherche, publiés ou non, émanant des établissements d'enseignement et de recherche français ou étrangers, des laboratoires publics ou privés. 


\section{Synergistic effect of the $\mathrm{TiCl}_{4} / p$-TsOH promoter system on the Aza-Prins cyclization}

Vianney Durel, Claudia Lalli,* Thierry Roisnel and Pierre van de Weghe*

Université de Rennes 1, UMR CNRS 6226, Institut des Sciences Chimiques de Rennes, Equipe PNSCM, UFR des Sciences Biologiques et Pharmaceutiques, 2 avenue du Prof Léon Bernard, F35043 Rennes Cedex, France

*E-mail: pierre.van-de-weghe@univ-rennes1.fr

*E-mail: claudia.lalli@univ-rennes1.fr

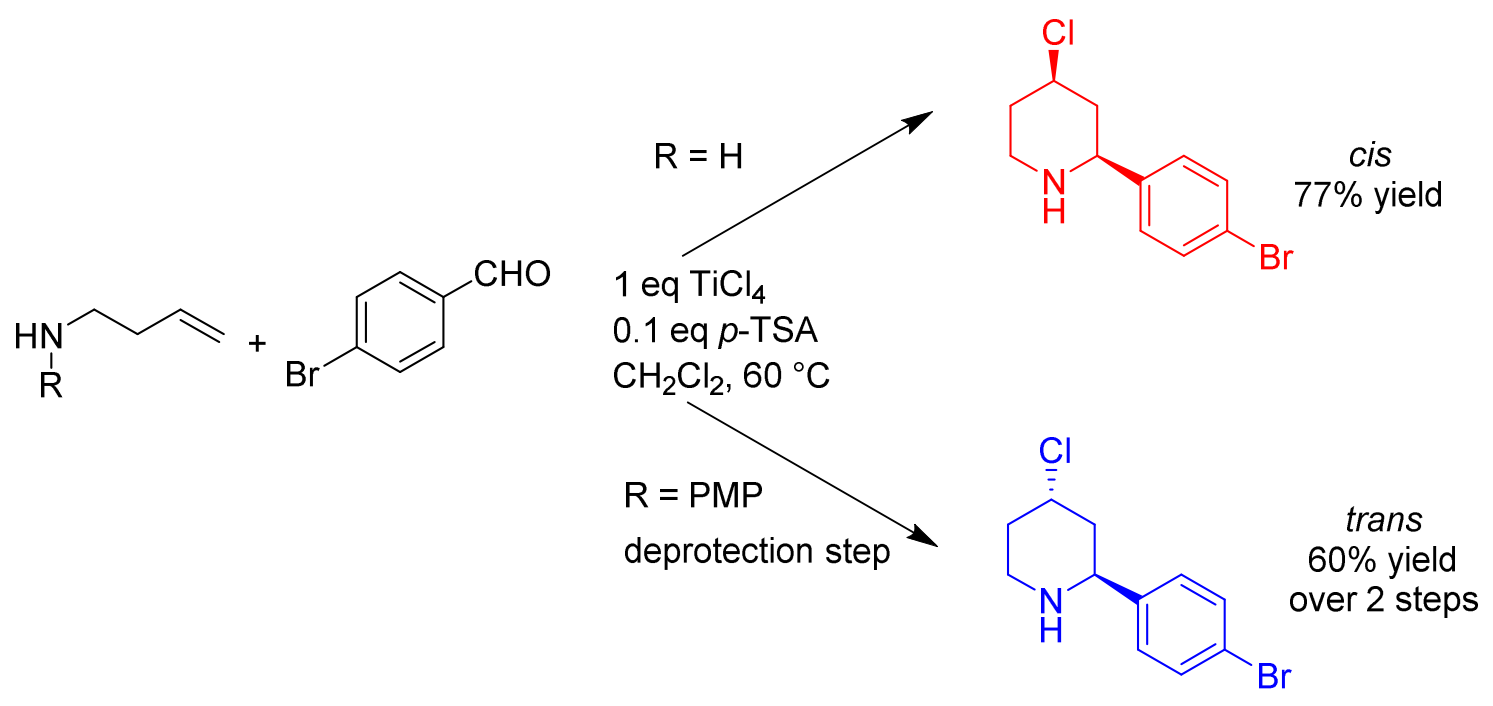


Abstract A novel aza-Prins cyclization promoted by a synergistic combination between a Lewis acid and a Brønsted acid to efficiently afford piperidines is described. Contrary to what has been previously reported in the literature, the generality of the reaction employing $N$-alkyl, $N$-aryl and non-protected homoallylamines has been demonstrated. The reaction is highly diastereoselective depending on the homoallylic amine used, $N$-PMP homoallyl amine leading preferentially to the trans diastereomer, and free homoallylamine affording the deprotected piperidine as single cis diastereomer.

\section{Introduction}

Nowadays there are many routes to prepare piperidines, among them the aza-Prins cyclization reaction involving aldehydes and homoallylic amines, appears to be a straightforward method that provides direct access to six-membered azacycles. ${ }^{1}$ Despite the great interest and the recent advances concerning the Prins cyclization, a feasible nitrogen-based version of this reaction knows only moderate success. Indeed this reaction is restricted in most cases to $\mathrm{N}$-sulfonyl homoallylic amines, which may limit the interest in this process and its development. Several Lewis acids are reported to promote the reaction of $\mathrm{N}$-sulfonyl homoallylic amines with aldehydes, among them, Fe(III) halides in stoichiometric or sub-stoichiometric amount, ${ }^{2} \mathrm{BiCl}_{3},{ }^{3} \mathrm{BF}_{3} \cdot \mathrm{Et}_{2} \mathrm{O},{ }^{4} \mathrm{InCl}_{3}{ }^{5}$ and TMSX. ${ }^{6}$ Brønsted acids are also reported to catalyze the cyclization, as in the case of phosphomolybdic acid, ${ }^{7} \mathrm{HBF}_{4} \cdot \mathrm{Et}_{2} \mathrm{O}^{8}$ and TfOH. ${ }^{9}$ It has to be pointed out that there are only a very few examples reported in the literature concerning aliphatic or primary amines. ${ }^{10}$ They concern mainly the use of the aza-Prins cyclization as key step in the total synthesis of biologically active alkaloids. To the best of our knowledge to date a detailed study devoted to the development of an effective aza-Prins cyclization involving nonsulfonylated homoallylic amines has not been undertaken. We wish herein to fill this gap by reporting our results on the aza-Prins cyclization reaction with $N$-aryl, -alkyl homoallylic amines and even with non-protected ones.

\section{Results}

We began our investigations by studying the reaction between $\mathrm{N}$-alkyl homoallylic amine as model substrate, namely $N$-methyl but-3-en-1-ylglycinate (1), and $p$-bromobenzaldehyde (2a), in $\mathrm{CH}_{2} \mathrm{Cl}_{2}$ at $60{ }^{\circ} \mathrm{C}$ in a sealed vial overnight (table 1). In the first attempts different Lewis acids known to promote 
the aza-Prins cyclization were screened, such as $\mathrm{FeCl}_{3}, \mathrm{AlCl}_{3}$ or $\mathrm{Bi}(\mathrm{OTf})_{3}$ (entries 1-3), unfortunately without any conversion of the starting materials, in some cases only the degradation of the reagents after a prolonged reaction time was observed. Only with 3 equiv. of $\mathrm{MgBr}_{2}$ or $\mathrm{TiCl}_{4}$ (entry 4-5) trace amounts of the desired product were detected in the crude NMR. The use of $p-\mathrm{TsOH} \cdot \mathrm{H}_{2} \mathrm{O}$, TFA and $p$ nitrobenzoic acid as Brønsted acids (entries 6-8) also failed to convert the starting materials into the desired product.

Table 1. Reaction conditions optimization. ${ }^{[a]}$

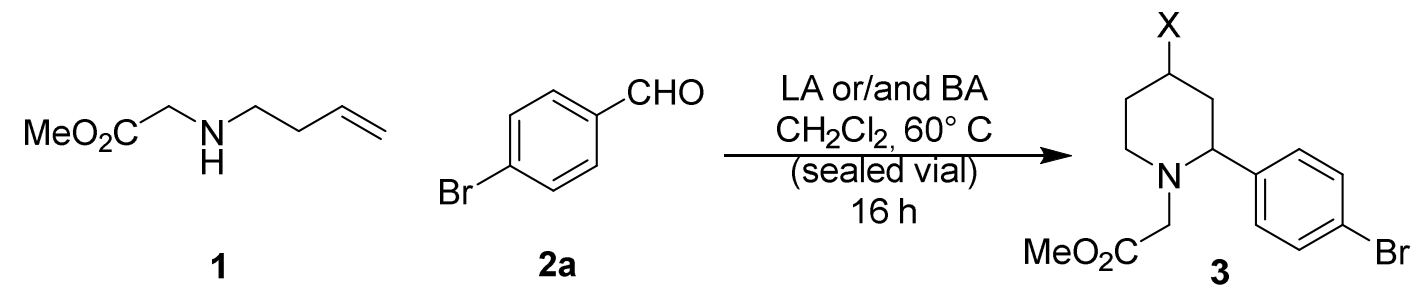

\begin{tabular}{|c|c|c|c|c|c|}
\hline Entry & $\begin{array}{l}\text { Lewis Acid } \\
\text { (equiv.) }\end{array}$ & $\begin{array}{l}\text { Brønsted Acid } \\
\text { (equiv.) }\end{array}$ & Yield $(\%)^{[\mathrm{b}]}$ & $\begin{array}{l}\text { Cis:trans } \\
\text { (d.r.) }^{[\mathrm{dd}]}\end{array}$ & $\mathrm{X}$ \\
\hline 1 & $\mathrm{FeCl}_{3}(3)$ & - & n.r. ${ }^{[c]}$ & - & - \\
\hline 2 & $\mathrm{AlCl}_{3}(3)$ & - & n.r. ${ }^{[\mathrm{c}]}$ & - & - \\
\hline 3 & $\mathrm{Bi}(\mathrm{OTf})_{3}(3)$ & - & n.r. ${ }^{[c]}$ & - & - \\
\hline 4 & $\mathrm{MgBr}_{2}(3)$ & - & traces & - & $\mathrm{Br}$ \\
\hline 5 & $\mathrm{TiCl}_{4}(3)$ & - & traces & - & $\mathrm{Cl}$ \\
\hline 6 & - & $p$-TsOH. $\mathrm{H}_{2} \mathrm{O}(3)$ & n.r. ${ }^{[\mathrm{c}]}$ & - & - \\
\hline 7 & - & TFA (3) & n.r. ${ }^{[\mathrm{c}]}$ & - & - \\
\hline 8 & - & $p-\mathrm{NO}_{2}-\mathrm{C}_{6} \mathrm{H}_{4} \mathrm{CO}_{2} \mathrm{H}$ & n.r. ${ }^{[\mathrm{c}]}$ & - & - \\
\hline 9 & $\mathrm{TiCl}_{4}$ & $p$-TsOH. $\mathrm{H}_{2} \mathrm{O}(1)$ & 98 & $57: 43$ & $\mathrm{Cl}$ \\
\hline 10 & $\mathrm{TiCl}_{4}(1)$ & $p$-TsOH. $\mathrm{H}_{2} \mathrm{O}(0.1)$ & 99 & $57: 43$ & $\mathrm{Cl}$ \\
\hline 11 & $\mathrm{MgBr}_{2}(1)$ & $p$-TsOH. $\mathrm{H}_{2} \mathrm{O}(0.1)$ & 55 & $50: 50$ & $\mathrm{Br}$ \\
\hline 12 & $\mathrm{AlCl}_{3}(1)$ & $p$-TsOH. $\mathrm{H}_{2} \mathrm{O}(0.1)$ & 43 & $45: 55$ & $\mathrm{Cl}$ \\
\hline 13 & $\mathrm{FeCl}_{3}(1)$ & $p$-TsOH. $\mathrm{H}_{2} \mathrm{O}(0.1)$ & 28 & $50: 50$ & $\mathrm{Cl}$ \\
\hline 14 & $\mathrm{ZnCl}_{2}(1)$ & $p$-TsOH. $\mathrm{H}_{2} \mathrm{O}(0.1)$ & 20 & $50: 50$ & $\mathrm{Cl}$ \\
\hline
\end{tabular}

[a] General conditions: 1 (1 equiv.), 2 a (1 equiv.), $\mathrm{CH}_{2} \mathrm{Cl}_{2}(0.05 \mathrm{M})$ at $60{ }^{\circ} \mathrm{C} 16 \mathrm{~h}$. [b] Yields refer to isolated products. [c] n.r.: no reaction. [d] ${ }^{1} \mathrm{H}$ NMR determination on the crude mixture. 
Within our recent investigations on the Prins cyclization, ${ }^{11}$ we reported a remarkable synergistic effect between non-reactive Brønsted and Lewis acids that lack the ability to catalyze the reaction if used alone. ${ }^{11 \mathrm{~b}}$ The benefit of this synergistic effect in the Prins cyclization was then confirmed when we disclosed the first enantioselective Prins cyclization by combining a chiral BINOL-derived bisphosphoric acid and $\mathrm{CuCl}^{11 \mathrm{c}}$

Based on our findings and on the previous observations by Aubé and coworkers, ${ }^{10 \mathrm{a}, \mathrm{b}}$ we decided to combine $\mathrm{TiCl}_{4}$ with $p$-TsOH. $\mathrm{H}_{2} \mathrm{O}$, the Lewis acid playing the role also of the nucleophile source. Thus 1, and 2a were reacted in the presence of 3 equiv. of $\mathrm{TiCl}_{4}$ and 1 equiv. of $p-\mathrm{TsOH} . \mathrm{H}_{2} \mathrm{O}$, in $\mathrm{CH}_{2} \mathrm{Cl}_{2}$ at $60{ }^{\circ} \mathrm{C}$ in a sealed vial overnight (table 1 , entry 9). To our delight the desired product was recovered in $98 \%$ yield, as a mixture of two diastereomers (cis:trans $=57: 43$ ). The variation of the Lewis and Brønsted acid amounts didn't affect the reactivity (see supporting information for details) and gratifyingly the expected piperidine was recovered in quantitative yield even with only 1 equiv. of $\mathrm{TiCl}_{4}$ and catalytic amount $(10 \mathrm{~mol} \%)$ of $p-\mathrm{TsOH} . \mathrm{H}_{2} \mathrm{O}$ and in the same diastereomeric ratio (table 1 , entry 10). A screening of different Brønsted and Lewis acid combinations revealed $\mathrm{TiCl}_{4}$ to be superior to $\mathrm{MgBr}_{2}, \mathrm{AlCl}_{3}, \mathrm{FeCl}_{3}$ and $\mathrm{ZnCl}_{2}$ (table 1, entries 11-14). On the contrary, the use of TFA, $\mathrm{MeSO}_{3} \mathrm{H}$, camphorsulfonic acid, as well as p-nitro benzoic acid with $\mathrm{TiCl}_{4}$ didn't affect the outcome of the reaction (see Supporting Information for details). Decreasing the reaction temperature to $40{ }^{\circ} \mathrm{C}$ was detrimental for the reactivity, moreover when the reaction was performed at $60{ }^{\circ} \mathrm{C}$ in $1,2-$ dichloroethane a slight drop of the yield was observed.

With the optimized conditions in hand we next examined the scope of the synergism between $\mathrm{TiCl}_{4}$ and $p$-TsOH. $\mathrm{H}_{2} \mathrm{O}$ with respect to different aldehydes, and the results are summarized in Table 2.

Table 2. Scope of the aza-Prins cyclization with the $N$-alkyl homoallylic amine 1 . $^{\text {a }}$

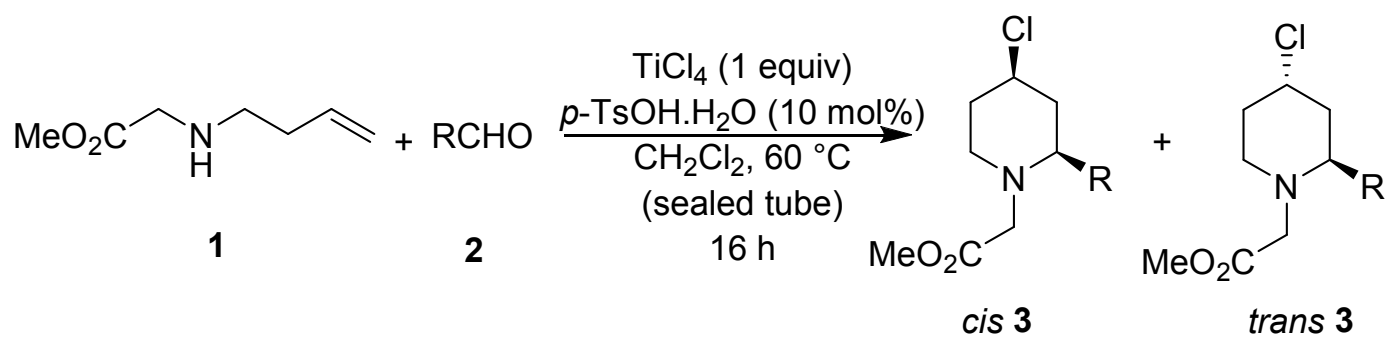




\begin{tabular}{|c|c|c|c|c|}
\hline Entry & Aldehyde 2 R= & Product 3 & Yield $(\%)^{[b]}$ & $\begin{array}{l}\text { Cis:trans } \\
\text { (d.r.) }^{[\mathrm{cc}]}\end{array}$ \\
\hline 1 & $p-\mathrm{Br}-\mathrm{C}_{6} \mathrm{H}_{4} \mathbf{2 a}$ & & 98 & $57: 43$ \\
\hline 2 & $\mathrm{C}_{6} \mathrm{H}_{5} \mathbf{2 b}$ & & 94 & $57: 43$ \\
\hline 3 & $o-\mathrm{Cl}-\mathrm{C}_{6} \mathrm{H}_{4} \mathbf{2 c}$ & & 86 & $86: 14$ \\
\hline 4 & $p-\mathrm{CN}-\mathrm{C}_{6} \mathrm{H}_{4} 2 \mathbf{d}$ & & 96 & $42: 58$ \\
\hline 5 & $p-\mathrm{NO}_{2}-\mathrm{C}_{6} \mathrm{H}_{4} \mathbf{2 e}$ & & 90 & $38: 62$ \\
\hline 6 & $m-\mathrm{NO}_{2}-\mathrm{C}_{6} \mathrm{H}_{4} \mathbf{2 f}$ & & 97 & $45: 55$ \\
\hline 7 & $p-\mathrm{N}\left(\mathrm{CH}_{3}\right)_{2}-\mathrm{C}_{6} \mathrm{H}_{4} \mathbf{2 g}$ & & 60 & $50: 50$ \\
\hline 8 & $2,5-\left(\mathrm{OCH}_{3}\right)_{2}-\mathrm{C}_{6} \mathrm{H}_{3} \mathbf{2 h}$ & & 92 & $87: 13$ \\
\hline 9 & $o-\mathrm{OCH}_{3}-\mathrm{C}_{6} \mathrm{H}_{4} 2 \mathbf{i}$ & & 65 & $85: 15$ \\
\hline 10 & $p-\mathrm{OCH}_{3}-\mathrm{C}_{6} \mathrm{H}_{4} \mathbf{2} \mathbf{j}$ & & $60^{[\mathrm{d}]}$ & $62: 38$ \\
\hline 11 & $p-\mathrm{CH}_{3}-\mathrm{C}_{6} \mathrm{H}_{4} 2 \mathbf{k}$ & & 74 & $62: 38$ \\
\hline 12 & $\left(\mathrm{CH}_{3}\right)_{2} \mathrm{CH} 2 \mathrm{l}$ & & 86 & $43: 57$ \\
\hline
\end{tabular}


$13^{[\mathrm{e}]} \quad \mathrm{C}_{6} \mathrm{H}_{4}-\left(\mathrm{CH}_{2}\right)_{2} 2 \mathbf{m}$

$14^{[\mathrm{e}]}\left(\mathrm{CH}_{3}\right)\left(\mathrm{CH}_{2}\right)_{6} \mathbf{2 n}$<smiles>CC(=O)N1CCC(Cl)C1CCc1ccc([Hg])cc1</smiles>

[a] General conditions: 1 (1 equiv.), 2 (1 equiv.), $\mathrm{TiCl}_{4}$ (1 equiv.), $p$-TsOH. $\mathrm{H}_{2} \mathrm{O}$ (10 mol\%), in $\mathrm{CH}_{2} \mathrm{Cl}_{2}$ $(0.1 \mathrm{M})$ at $60{ }^{\circ} \mathrm{C}$ in a sealed vial $16 \mathrm{~h}$. [b] Yields refer to isolated products. [c] ${ }^{1} \mathrm{H}$ NMR determination on the crude mixture. [d] 62\% conversion. [e] Performed with 2 (1.5 equiv).

Gratifyingly benzaldehyde $\mathbf{2 b}$ (entry 2 ) smoothly participated to the reaction leading to the desired product $\mathbf{3 b}$ in $94 \%$ yield, and the same cis:trans selectivity (d.r. 57:43). The aromatic ring substitution in the ortho, meta or para position with electron-withdrawing groups (entries 3-6) is well tolerated as in all the cases the piperidines 3c-f were obtained in $86-97 \%$ yields. The reaction proved to be less efficient for aromatic aldehydes substituted with an electron-donating group especially in the para position (entries 7, 10 and 11), leading to the products $\mathbf{3 g}, \mathbf{j}$ and $\mathbf{k}$ in $60 \%, 60 \%$ and $74 \%$ yield respectively. It's interesting to note that in the presence of an ortho substituent on the aromatic ring, the cis-product was obtained as major diastereomer (entries 3,8 and 9) affording the desired piperidines with up to $87: 13$ d.r. probably because of the steric hindrance. ${ }^{12}$ Aliphatic aldehydes undergo aza-Prins cyclization in high yields (entries 12-14), however in the case of 3phenylpropionaldehyde (2m) and $n$-octanal (2n) a slight excess of aldehyde was needed (1.5 equiv) to have a complete conversion of $\mathbf{1}$; these aldehydes reacting with themselves leading to small amount of the aldol-crotonization products.

The scope of the reaction was then extended to other protected homoallylic amines. Contrarily to the scarce previously reported results, ${ }^{13,3,10 \mathrm{c}}$ our reaction conditions are not compatible with the use of carbamates as protecting group on the nitrogen atom. Both the Boc- and Cbz- are cleaved in the presence of the combination of $\mathrm{TiCl}_{4}$ and $p$-TsOH. $\mathrm{H}_{2} \mathrm{O}$ and no trace of the aza-Prins product was observed. Otherwise the aza-Prins reaction with homoallylic amine protected by the widely used $p$ methoxyphenyl (PMP) group 4 was also carried out and the results are reported in Table 3.

Table 3. Scope of the aza-Prins cyclization with PMP-protected homoallylic amine 4. ${ }^{\mathrm{a}}$ 
<smiles>[R]C=[C+]=CCCN[Y16]</smiles>

4

$\mathrm{PMP}=p-\mathrm{OMe}-\mathrm{Ph}$

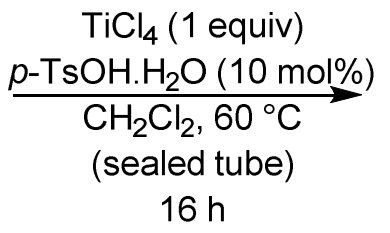

$16 \mathrm{~h}$<smiles>[R]C1C[C+](Cl)CCN1[R16]</smiles>

cis 5

trans 5

\begin{tabular}{|c|c|c|c|c|}
\hline Entry & Aldehyde 2 R= & Product 5 & Yield $(\%)^{[b]}$ & $\begin{array}{l}\text { Cis/trans } \\
\text { (d.r.) }{ }^{[\mathrm{c}]}\end{array}$ \\
\hline 1 & $p-\mathrm{Br}-\mathrm{C}_{6} \mathrm{H}_{4} \mathbf{2 a}$ & & 99 & $12: 88$ \\
\hline 2 & $\mathrm{C}_{6} \mathrm{H}_{5} \mathbf{2 b}$ & & 93 & $23: 77$ \\
\hline 3 & $o-\mathrm{Cl}-\mathrm{C}_{6} \mathrm{H}_{4} 2 \mathrm{c}$ & & 99 & $28: 72$ \\
\hline 4 & $p-\mathrm{NO}_{2}-\mathrm{C}_{6} \mathrm{H}_{4} \mathbf{2 e}$ & & 92 & $14: 86$ \\
\hline 5 & $p-\mathrm{OCH}_{3}-\mathrm{C}_{6} \mathrm{H}_{4} \mathbf{2} \mathbf{j}$ & & $40^{[\mathrm{d}]}$ & $20: 80$ \\
\hline 6 & $p-\mathrm{CH}_{3}-\mathrm{C}_{6} \mathrm{H}_{4} \mathbf{2 k}$ & & 90 & $22: 78$ \\
\hline 7 & $\left(\mathrm{CH}_{3}\right)_{2} \mathrm{CH} 2 \mathrm{I}$ & & 98 & 9:91 \\
\hline
\end{tabular}

[a] General conditions: 4 (1 equiv.), 2 (1 equiv.), $\mathrm{TiCl}_{4}$ (1 equiv.), $p$-TsOH.H $\mathrm{H}_{2} \mathrm{O}(10 \mathrm{~mol} \%)$, in $\mathrm{CH}_{2} \mathrm{Cl}_{2}$ $(0.1 \mathrm{M})$ at $60{ }^{\circ} \mathrm{C}$ in a sealed vial $16 \mathrm{~h}$. [b] Yields refer to isolated products. [c] ${ }^{1} \mathrm{H}$ NMR determination on the crude mixture. [d] $49 \%$ conversion.

In the presence of aromatic aldehydes substituted on the ortho or para positions with electron withdrawing groups 2a-c and e the aza-Prins products 5a-d, were isolated in up to $99 \%$ yields, this time surprisingly, with a good diastereoselection in favor of the trans isomer (d.r. up to 86:14). Aliphatic isobutyraldehyde $\mathbf{2} \mathbf{l}$ also provided the desired piperidine $\mathbf{5 g}$ in very good yield and as single diastereomer (trans:cis $=91: 9$ ). The substitution on the aromatic ring with strong electron donating groups is less tolerated. Indeed, while p-tolualdehyde (2k) smoothly reacted to give the desired 
piperidine in $90 \%$ yield (entry 6), $p$-anisaldehyde (2j) gave the product in only $40 \%$ yield (entry 5) and 2,5-dimethoxy benzaldehyde (2h) did not react at all. This is probably due to electronic effects, since the iminium intermediate is too electron enriched due to the donating PMP and methoxy groups. To illustrate the synthetic utility of this methodology we deprotected the PMP group in standard reaction conditions, in the presence of ceric ammonium nitrate $\mathrm{CAN}$, in order to obtain the $\mathrm{NH}$ free piperidine as the single trans 7a diastereomer in $73 \%$ yield (Scheme 1), thus with an overall yield of $66 \%$ over two steps.

Scheme 1. Deprotection of the PMP group of the piperidine trans $5 \mathbf{a}$.

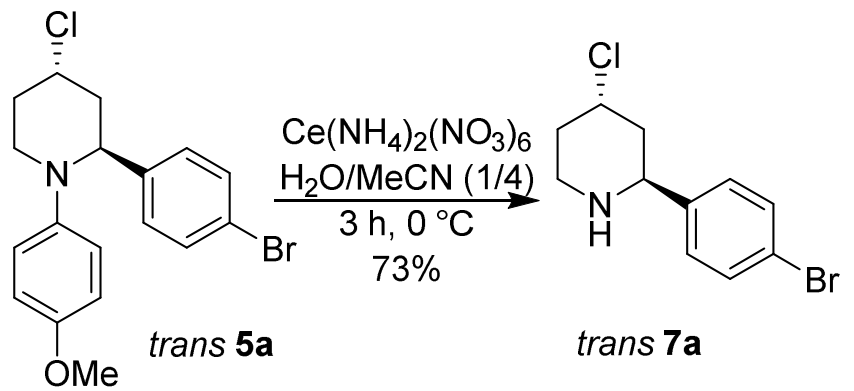

We next wondered about the possibility to carry out the aza-Prins cyclization with the but-3-en-1amine (6). Indeed very few examples are reported in the literature using a free homoallyl amine or an imine. To our delight, although the reaction time was longer ( $90 \mathrm{~h}$ instead of overnight) the synergistic combination of $\mathrm{TiCl}_{4}$ with $p$-TsOH. $\mathrm{H}_{2} \mathrm{O}$ allowed isolation of the expected piperidines $\mathbf{7}$ in good yields and gratifyingly as the single cis-stereoisomer (d.r. > 95/5) (Table 4).

Table 4. Scope of the aza-Prins yclization with homoallyl amine $6{ }^{a}$

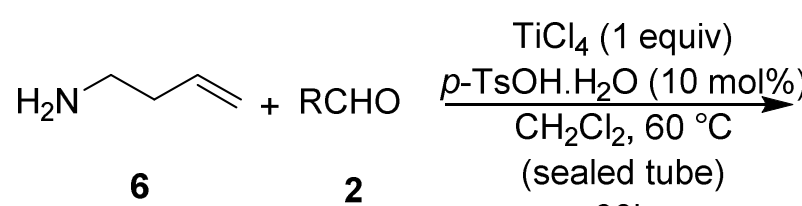

90h<smiles>[R]C1C[C@@H](Cl)CCN1</smiles>

cis 7

d.r. $>95: 5$

\begin{tabular}{llll}
\hline Entry & Aldehyde $\mathbf{2} \mathrm{R}=$ & Product 7 & ${\text { Yield }(\%)^{[\mathrm{b}]}}$ \\
\hline 1 & $p-\mathrm{Br}^{-} \mathrm{C}_{6} \mathrm{H}_{4} \mathbf{2 a}$ &
\end{tabular}


2

$\mathrm{C}_{6} \mathrm{H}_{5} \mathbf{2 b}$

3

$o-\mathrm{Cl}-\mathrm{C}_{6} \mathrm{H}_{4} 2 \mathrm{c}$

4

$p-\mathrm{NO}_{2}-\mathrm{C}_{6} \mathrm{H}_{4} \mathbf{2 e}$

5

$p-\mathrm{OCH}_{3}-\mathrm{C}_{6} \mathrm{H}_{4} \mathbf{2} \mathbf{j}$

6

$p-\mathrm{CH}_{3}-\mathrm{C}_{6} \mathrm{H}_{4} \mathbf{2 k}$

7 $\sum_{7 b}^{C l}$

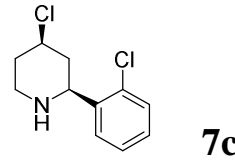

$\overbrace{\mathrm{NO}_{2} 7 \mathbf{7}}^{\mathrm{Cl}}$
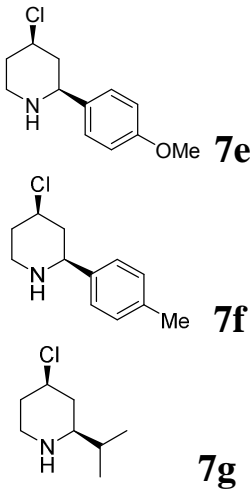

82

$34^{[\mathrm{c}]}$

86

80

$70^{[\mathrm{c}]}$

88

[a] General conditions: 6 (1 equiv.), 2 (1 equiv.), $\mathrm{TiCl}_{4}$ (1 equiv.), $p$-TsOH. $\mathrm{H}_{2} \mathrm{O}$ (10 mol\%), in $\mathrm{CH}_{2} \mathrm{Cl}_{2}$ $(0.1 \mathrm{M})$ at $60{ }^{\circ} \mathrm{C}$ in a sealed vial $90 \mathrm{~h}$. [b] Yields refer to isolated products. [c] Reaction time prolonged to $160 \mathrm{~h}$.

Again the only exception in this trend concerns the electron rich aldehydes; the reaction with $\mathbf{2 k}$ afforded the piperidine $\mathbf{7 f}$ in $70 \%$ yield after a longer reaction time $(160 \mathrm{~h})$ and $\mathbf{2} \mathbf{j}$ afforded $\mathbf{7 g}$ in only $32 \%$ yield after $160 \mathrm{~h}$.

The structure of compounds trans $\mathbf{3 e}$ and cis $\mathbf{7 a}$ and their relative configurations were determined by NOESY experiments ${ }^{14}$ and confirmed by single crystal X-ray analysis ${ }^{14}$ (Figure 1). 
Figure 1 ORTEP representation of the piperidines products trans-3e and cis-7a. The thermal ellipsoids are shown at $50 \%$ probability.<smiles>CC(=O)CN1CC[C@H](Cl)C[C@H]1c1ccc([N+](=O)[O-])cc1</smiles>

trans $3 \mathrm{e}$<smiles>ClC1CCN[C@H](c2ccc(Br)cc2)C1</smiles>

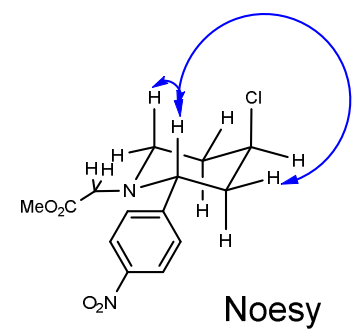

Noesy
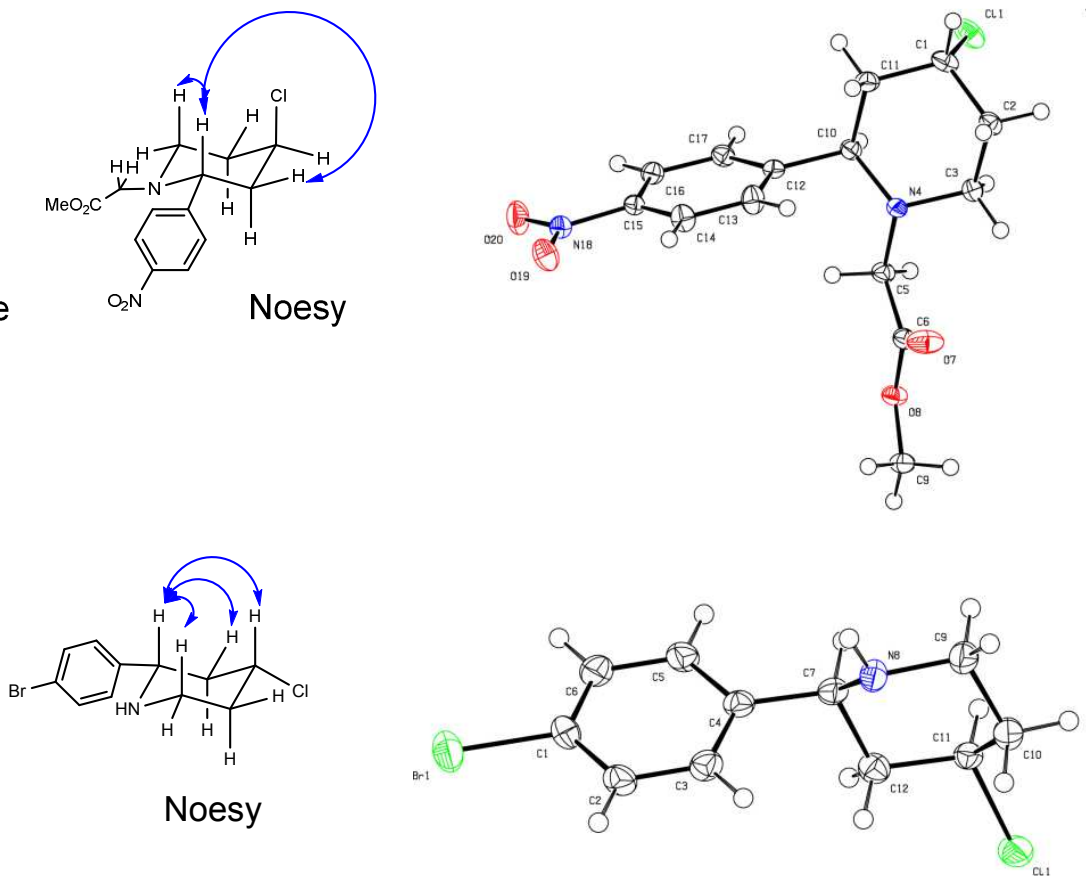

It is worth noting that the cis/trans ratio is strongly dependent on the feature of the nitrogen atom substituent, as depicted in Table 5. To determine the trend of the selectivity dependence, the reactions between benzaldehyde (2b) and different substituted homoallylic amines were performed.

Table 5. Selectivity dependence.

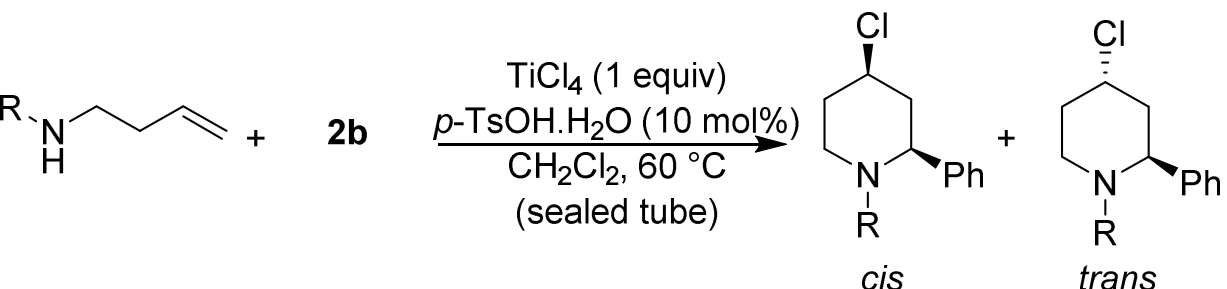

\begin{tabular}{|c|c|c|c|c|}
\hline Entry & \multicolumn{2}{|l|}{$\mathrm{R}=$} & Product (Yield \%) & 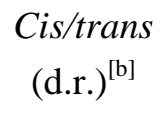 \\
\hline 1 & \multicolumn{2}{|c|}{$\mathrm{CH}_{2} \mathrm{CO}_{2} \mathrm{Me}(\mathbf{1})$} & $\mathbf{3 b}(94)$ & $57: 43$ \\
\hline 2 & PMP & (4) & $\mathbf{5 b}(93)$ & $23: 77$ \\
\hline 3 & $\mathrm{H}$ & (6) & $7 b(86)$ & $>95: 5$ \\
\hline 4 & Ts & (8) & $11(93)$ & $>5: 95$ \\
\hline 5 & $\mathrm{Bn}$ & (9) & $12(66)$ & $50: 50$ \\
\hline 6 & $n$-Pr & (10) & $13(90)$ & $50: 50$ \\
\hline
\end{tabular}

[a] Yields refer to isolated products. [b] ${ }^{1} \mathrm{H}$ NMR determination on the crude mixture. 
With $N$-tosyl homoallyl amine (8) only the product trans $\mathbf{1 1}$ was recovered in $95 \%$ yield (entry 4 ). Next we employed $N$-alkyl homoallylic amines such as $N$-benzyl (9) and $N$-propyl (10) (entries 5 and 6): both delivered an equimolar mixture of cis and trans isomers $\mathbf{1 2}$ and $\mathbf{1 3}$ in good yield. We can therefore conclude that whereas the free amine $\mathbf{6}$ led to the piperidine cis $\mathbf{7 b}$ as unique stereoisomer (entry 3), the arylamine $\mathbf{4}$ and the tosylamine $\mathbf{8}$ gave the trans isomer as major product (entries 2 and 4), finally no selectivity was observed with the alkylamines $\mathbf{1 , 9}$ and $\mathbf{1 0}$ (entries 1,5 and 6).

These results could be explained as follow (Figure 2). According to the common reaction mechanism of the aza-Prins cyclization, the reaction starts by the formation of an iminium whose $\mathbf{A}$ and $\mathbf{B}$ forms are in equilibrium and then the nucleophilic attack (here the chloride anion) occurs on the equatorial position. As mentioned by Padrón et al., ${ }^{2 \mathrm{a}}$ in the case of $\mathrm{R}^{1}=\mathrm{Ts}$, ab-initio calculations showed that the iminium $\mathbf{B}$ is more stable than the isomer $\mathbf{A}$. This is probably due to the strong steric repulsion between the groups $R^{1}$ and $R$ in comparison to the lower energy cost of the allylic strain between $R$ and $\mathrm{H}$. Obviously, this comment can also explain the trans- selectivity with $\mathrm{R}^{1}=\mathrm{PMP}$. When $\mathrm{R}^{1}=\mathrm{H}$, only the allylic strain remains, ${ }^{15}$ favoring thus the formation of the iminium $\mathbf{A}$ and consequently the formation of the cis-piperidine. The observed lack of selectivity when the amine bears an alkyl chain (homoallylic amine 1) is certainly due to an equivalent energy cost between the steric repulsion for the iminium $\mathbf{A}$ and the allylic strain for $\mathbf{B}$. This last case helps us to better understand the high cisselectivity when the reaction was carried out with ortho-substituted aryl aldehydes (Table 2, entries 38-9). These ortho-substituents could contribute to increase the steric hindrance detrimental to the iminium $\mathbf{B}$ by reinforcing the negative contribution of the allylic strain versus the steric repulsion between the residue $\mathrm{CH}_{2} \mathrm{CO}_{2} \mathrm{Me}\left(\mathrm{R}^{1}\right)$ and the aryl group (R).

Figure 2. Comparison of possible transition structures for the cyclization reaction. 


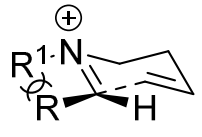

iminium A<smiles>[CH]=C</smiles>

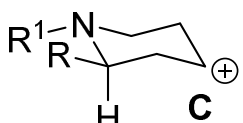

$\downarrow$

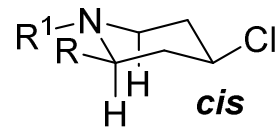

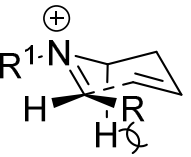

iminium B<smiles>C=C</smiles>

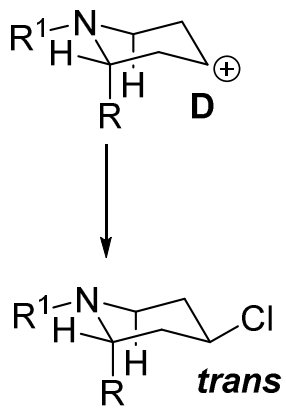

In conclusion, we have demonstrated that the synergism between $\mathrm{TiCl}_{4}$ and $p$ - $\mathrm{TsOH} . \mathrm{H}_{2} \mathrm{O}$ can promote the aza-Prins cyclization with $N$-aryl, -alkyl and even non protected homoallylic amines. The piperidine derivatives are obtained in good yields and with a cis/trans ratio dependent on the group borne by the nitrogen atom. The trans-isomer was obtained as major compound when tosyl and PMP are used as protecting groups, while the cis-isomer was formed in the absence of protecting group. This methodology can be useful for preparing either cis- or trans- piperidines and could be later used for the synthesis of valuable piperidine scaffolds found in natural and bioactive products.

\section{Experimental section}

\section{General information}

All the reactions were performed in dried glassware, under argon atmosphere and with dry solvents. Reagents were obtained from commercial suppliers and used without further purification unless otherwise noted. TLC analyses were performed using precoated Merck TLC Silica Gel 60 F254 plates. Purifications by column chromatography on silica gel were performed using Merck Silica Gel 60 (70230 mesh) and purifications by preparative thin layer chromatography on silica gel using Merck Silica Gel 60 PF254. Petroleum ether (PE) used for purifications was the low boiling point fraction (40-60 $\left.{ }^{\circ} \mathrm{C}\right) .{ }^{1} \mathrm{H}$ NMR and ${ }^{13} \mathrm{C}$ spectra were recorded on a $300 \mathrm{Mhz}$ instrument using TMS and $\mathrm{CDCl}_{3}$ respectively as internal standards. Chemical shifts $(\delta)$ are reported in parts per million (ppm). The 
following abbreviations are used for multiplicities: s, singlet; $d$, doublet; $t$, triplet; dd, doublet of doublets; q, quadruplet; quint, quintuplet; td, triplet of doublets; dt, doublet of triplets; tt, triplet of triplets, m, multiplet. Carbon multiplicities were determined by Jmod experiments. Coupling constants $(J)$ are reported in Hertz (Hz). - HRMS analyses were obtained using MaXis 4G or a TOF Q for ESI. X-ray crystallographic data were collected on a crystal diffractometer. Melting points were obtained on a hot bench.

Preparation of $N$-methyl but-3-en-1-ylglycinate (1): To a solution of methyl glycinate hydrochloride (11.12 g, 2 equiv., $88.6 \mathrm{mmol})$ in acetonitrile $(220 \mathrm{~mL})$ was added $\mathrm{K}_{2} \mathrm{CO}_{3}(18.36 \mathrm{~g}, 3$ equiv., $133 \mathrm{mmol})$. The mixture was stirred $1 \mathrm{~h}$ at room temperature, then 4-bromo-1-butene $(4.5 \mathrm{~mL}$, 1 equiv., $44.9 \mathrm{mmol}$ ) was added and the stirring continued at $45{ }^{\circ} \mathrm{C}$ for $48 \mathrm{~h}$. The insoluble material was filtered off and the filtrate concentrated under reduced pressure. $\mathrm{CH}_{2} \mathrm{Cl}_{2}(60 \mathrm{~mL})$ and $\mathrm{H}_{2} \mathrm{O}$ were added, the two-phase mixture was separated, and the aqueous phase was extracted twice with $\mathrm{CH}_{2} \mathrm{Cl}_{2}$. The combined organic phases were dried over $\mathrm{MgSO}_{4}$, filtered, and evaporated in vacuo. Compound 1 was isolated as colorless oil $(6.01 \mathrm{~g}, 90 \%$ yield $)$ after distillation $\left(70{ }^{\circ} \mathrm{C}\right.$, to reduced pressure $) .{ }^{1} \mathrm{H}$ NMR $\left(\mathrm{CDCl}_{3}, 300 \mathrm{MHz}\right): \delta=5.85-5.75(\mathrm{~m}, 1 \mathrm{H}), 5.14-5.04(\mathrm{~m}, 2 \mathrm{H}), 3.73(\mathrm{~s}, 3 \mathrm{H}), 3.43(\mathrm{~s}, 2 \mathrm{H}), 2.68$ $(\mathrm{t}, J=6.7 \mathrm{~Hz}, 2 \mathrm{H}), 2.26(\mathrm{q}, J=6.7 \mathrm{~Hz}, 2 \mathrm{H}), 1.66(\mathrm{bs}, \mathrm{NH}) .{ }^{13} \mathrm{C} \mathrm{NMR}\left(\mathrm{CDCl}_{3}, 75 \mathrm{MHz}\right): \delta=173.0$ $(\mathrm{C}=\mathrm{O}), 136.2(=\mathrm{CH}), 116.7\left(=\mathrm{CH}_{2}\right), 51.9\left(\mathrm{CH}_{3}\right), 50.8\left(\mathrm{CH}_{2}\right), 48.6\left(\mathrm{CH}_{2}\right), 34.4\left(\mathrm{CH}_{2}\right)$. ESI-HRMS calculated for $\mathrm{C}_{7} \mathrm{H}_{14} \mathrm{NO}_{2}[\mathrm{M}+\mathrm{H}]^{+}$144.1024, found 144.1022.

Preparation of $N$-(but-3-en-1-yl)-4-methoxyaniline (4): To a solution of $p$-anisidine $(6.16 \mathrm{~g}, 5$ equiv., $49.2 \mathrm{mmol}$ ) and 4-bromo-1-butene (1.33 g, 1 equiv., $9.85 \mathrm{mmol})$ in EtOH (20 mL) was added $\mathrm{NaI}$ (147 mg, 0.1 equiv., $0.98 \mathrm{mmol})$. The mixture was stirred to reflux for $4 \mathrm{~h}$, and then the solvent was removed in vacuo. $\mathrm{CH}_{2} \mathrm{Cl}_{2}(20 \mathrm{~mL})$ followed by $\mathrm{KOH}(1 \mathrm{M}, 20 \mathrm{~mL})$ were added. The two-phase mixture was separated, and the organic phase was washed with water $(2 \times 20 \mathrm{~mL})$ and brine $(2 \times 20$ $\mathrm{mL}$ ), dried over $\mathrm{MgSO}_{4}$, filtered, and evaporated in vacuo. The compound 4 was isolated as brown oil (1.58 g, 90\% yield) after purification by flash chromatography (10\% EtOAc in petroleum ether). ${ }^{1} \mathrm{H}$ NMR $\left(\mathrm{CDCl}_{3}, 300 \mathrm{MHz}\right): \delta=6.78(\mathrm{~d}, J=8.8 \mathrm{~Hz} 2 \mathrm{H}), 6.58(\mathrm{~d}, J=8.8 \mathrm{~Hz}, 2 \mathrm{H}), 5.86-5.75(\mathrm{~m}, 1 \mathrm{H})$, 5.16-5.08 (m, 2 H), 3.73 (s, $3 \mathrm{H}), 3.27$ (bs, NH), 3.13 (t, $J=6.7 \mathrm{~Hz}, 2 \mathrm{H}), 2.36$ (q, $J=6.7 \mathrm{~Hz}, 2 \mathrm{H})$. 
${ }^{13} \mathrm{C} \mathrm{NMR}\left(\mathrm{CDCl}_{3}, 75 \mathrm{MHz}\right): \delta=152.2(\mathrm{C}), 142.6(\mathrm{C}), 136.0(=\mathrm{CH}), 117.1\left(=\mathrm{CH}_{2}\right), 115.0(2 \times \mathrm{CH})$, $114.4(2 \times \mathrm{CH}), 55.9\left(\mathrm{CH}_{3}\right), 43.9\left(\mathrm{CH}_{2}\right), 33.8\left(\mathrm{CH}_{2}\right)$. ESI-HRMS calculated for $\mathrm{C}_{11} \mathrm{H}_{16} \mathrm{NO}[\mathrm{M}+\mathrm{H}]^{+}$ 178.1232, found 178.1232 .

Preparation of $\mathrm{N}$-but-3-en-1-yl-4-methylbenzenesulfonamide (8): This substrate was synthesized according to a described procedure. ${ }^{16}$ To a solution of 3-buten-1-amine (662 mg, 1 equiv., $9.3 \mathrm{mmol}$ ), $\mathrm{NEt}_{3}(1.9 \mathrm{~mL}, 1.5$ equiv., $13.9 \mathrm{mmol})$ and dimethylaminopyridine $(341 \mathrm{mg}, 0.3$ equiv., $2.79 \mathrm{mmol})$ in $\mathrm{CH}_{2} \mathrm{Cl}_{2}(30 \mathrm{~mL})$, at $0{ }^{\circ} \mathrm{C}$ tosyl chloride $(2.13 \mathrm{~g}, 11.2 \mathrm{mmol}, 1.2 \mathrm{eq})$ was added. The reaction was warmed to r.t., stirred for $3 \mathrm{~h}$, quenched with water and the aqueous layer was extracted three times with $\mathrm{CH}_{2} \mathrm{Cl}_{2}$. The combined organic phases were dried over $\mathrm{MgSO}_{4}$, filtered, and evaporated in vacuo. The residue was purified by flash chromatography (EP/EtOAc 4:1) to afford the corresponding tosylamine $15(2.08 \mathrm{~g}, 99 \%$ yield $)$ as colourless oil. ${ }^{1} \mathrm{H} \mathrm{NMR}\left(\mathrm{CDCl}_{3}, 300 \mathrm{MHz}\right): \delta=7.75(\mathrm{~d}, J=8.4$ $\mathrm{Hz}, 2 \mathrm{H}), 7.32(\mathrm{~d}, J=8.4 \mathrm{~Hz} 2 \mathrm{H}), 5.69-5.56(\mathrm{~m}, 1 \mathrm{H}), 5.09-5.01(\mathrm{~m}, 2 \mathrm{H}), 4.42(\mathrm{~s}, \mathrm{NH}), 3.02(\mathrm{q} J=$ $6.6 \mathrm{~Hz}, 2 \mathrm{H}), 2.43(\mathrm{~s}, 3 \mathrm{H}), 2.20(\mathrm{q}, J=6.6 \mathrm{~Hz}, 2 \mathrm{H}) .{ }^{13} \mathrm{C} \mathrm{NMR}\left(\mathrm{CDCl}_{3}, 75 \mathrm{MHz}\right): \delta=143.6(\mathrm{C})$, $137.1(\mathrm{C}), 134.3(=\mathrm{CH}), 129.9(2 \times \mathrm{CH}), 127.3(2 \times \mathrm{CH}), 118.4\left(=\mathrm{CH}_{2}\right), 42.2\left(\mathrm{CH}_{2}\right), 33.7\left(\mathrm{CH}_{2}\right) 21.7$ $\left(\mathrm{CH}_{3}\right)$.

Preparation of $N$-benzylbut-3-en-1-amine (9): This substrate was synthesized according to a described procedure. ${ }^{17}$ To a solution of benzylamine (5.25 g, 5 equiv., $49.0 \mathrm{mmol}$ ) and 4-bromo-1butene ( $1 \mathrm{~mL}, 1$ equiv., $9.85 \mathrm{mmol})$ in $\mathrm{EtOH}(20 \mathrm{~mL}), \mathrm{NaI}(150 \mathrm{mg}, 0.1$ equiv., $0.98 \mathrm{mmol})$ was added. The mixture was stirred to reflux for $4 \mathrm{~h}$. Then the solvent was removed in vacuo, $\mathrm{CH}_{2} \mathrm{Cl}_{2}(20$ $\mathrm{mL})$ followed by $\mathrm{KOH}(1 \mathrm{M}, 20 \mathrm{~mL})$ were added and the two-phase mixture was separated. The organic phase was washed with water $(2 \times 20 \mathrm{~mL})$ and brine $(2 \times 20 \mathrm{~mL})$, dried over $\mathrm{MgSO}_{4}$, filtered, and evaporated in vacuo. The residue was purified by flash chromatography $(10 \%$ EtOAc in petroleum ether) to give pure product 17 ( $N$-benzylbut-3-en-1-amine) as a yellow oil $(1.51 \mathrm{~g}, 95 \%$ yield). ${ }^{1} \mathrm{H} \mathrm{NMR}\left(\mathrm{CDCl}_{3}, 300 \mathrm{MHz}\right): \delta=7.33-7.22(\mathrm{~m}, 5 \mathrm{H}), 5.85-5.72(\mathrm{~m}, 1 \mathrm{H}), 5.12-5.02(\mathrm{~m}, 2 \mathrm{H})$, $3.81(\mathrm{~s}, 2 \mathrm{H}), 2.71(\mathrm{t}, J=6.8 \mathrm{~Hz}, 2 \mathrm{H}), 2.31(\mathrm{qt}, J=6.8,1.3 \mathrm{~Hz}, 2 \mathrm{H}), 1.84(\mathrm{br} \mathrm{s}, 1 \mathrm{H}) .{ }^{13} \mathrm{C} \mathrm{NMR}$ $\left(\mathrm{CDCl}_{3}, 75 \mathrm{MHz}\right): \delta=140.1(\mathrm{C}), 136.5(=\mathrm{CH}), 128.6(2 \times \mathrm{CH}), 128.3(2 \times \mathrm{CH}), 127.1(\mathrm{CH}), 116.6$ $\left(=\mathrm{CH}_{2}\right), 53.9\left(\mathrm{CH}_{2}\right), 48.3\left(\mathrm{CH}_{2}\right), 34.3\left(\mathrm{CH}_{2}\right)$. 
Preparation of $N$-propylbut-3-en-1-amine (10): To a solution of but-3-en-1-amine $(0.77 \mathrm{~mL}, 1.0$ equiv., $8.4 \mathrm{mmol})$ in $\mathrm{MeOH}(10 \mathrm{~mL})$ was added propanal $(0.74 \mathrm{~mL}, 1.2$ equiv., $10.1 \mathrm{mmol})$. The mixture was stirred at room temperature for $2 \mathrm{~h}$. Solid $\mathrm{NaBH}_{4}$ (794 mg, 2.5 equiv., $21 \mathrm{mmol}$ ) was then added portionwise over $1 \mathrm{~h}$. The reaction was stirred at room temperature overnight, quenched with 2.0 $\mathrm{M}$ aq. $\mathrm{NaOH}(10 \mathrm{~mL})$ and extracted with $\mathrm{Et}_{2} \mathrm{O}(3 \times 30 \mathrm{~mL})$. The organic layers were combined, dried over $\mathrm{MgSO}_{4}$, filtered, and evaporated in vacuo. The residue was purified by distillation $\left(70{ }^{\circ} \mathrm{C}\right.$ under reduce pressure) to give compound 19 (900 mg, 95\% yield) as a colorless oil. ${ }^{1} \mathrm{H}$ NMR $\left(\mathrm{CDCl}_{3}\right.$, $300 \mathrm{MHz}): \delta=5.86-5.72(\mathrm{~m}, 1 \mathrm{H}), 5.12-5.02(\mathrm{~m}, 2 \mathrm{H}), 2.67(\mathrm{t}, J=6.8 \mathrm{~Hz}, 2 \mathrm{H}), 2.57(\mathrm{t}, J=7.3 \mathrm{~Hz}, 2$ $\mathrm{H}), 2.28(\mathrm{qt}, J=6.8,1.2 \mathrm{~Hz}, 2 \mathrm{H}), 1.51(\mathrm{q}, J=7.3 \mathrm{~Hz}, 2 \mathrm{H}), 0.91(\mathrm{t}, J=7.3 \mathrm{~Hz}, 3 \mathrm{H}) .{ }^{13} \mathrm{C} \mathrm{NMR}$ $\left(\mathrm{CDCl}_{3}, 75 \mathrm{MHz}\right): \delta=136.7(=\mathrm{CH}), 116.4\left(=\mathrm{CH}_{2}\right), 51.9\left(\mathrm{CH}_{2}\right), 48.9\left(\mathrm{CH}_{2}\right), 34.5\left(\mathrm{CH}_{2}\right), 23.3\left(\mathrm{CH}_{2}\right)$, $11.9\left(\mathrm{CH}_{3}\right)$.

4-Methoxyphenyl deprotection: To a solution of trans 5a (114.2 mg, 1.0 equiv., $0.3 \mathrm{mmol})$ in $\mathrm{MeCN}-\mathrm{H}_{2} \mathrm{O}(4: 1,6 \mathrm{~mL})$ was added ceric ammonium nitrate $\mathrm{Ce}\left(\mathrm{NH}_{4}\right)_{2}\left(\mathrm{NO}_{3}\right)_{6}(987 \mathrm{mg}, 6$ equiv., 1.8 mmol) at $0{ }^{\circ} \mathrm{C}$. The mixture was stirred at the same temperature for $3 \mathrm{~h}$. Solid $\mathrm{NaBH}_{4}(794 \mathrm{mg}, 2.5$ equiv., $21 \mathrm{mmol})$ was then added portionwise over $1 \mathrm{~h}$. Then water $(12 \mathrm{~mL})$ was added and extracted with EtOAc $(90 \mathrm{~mL})$. The aqueous layer was basified with $\mathrm{K}_{2} \mathrm{CO}_{3}$, filtered through a pad of celite and extracted with EtOAc $(2 \times 30 \mathrm{~mL})$. The organic layers were combined, dried over $\mathrm{MgSO}_{4}$, filtered, and evaporated in vacuo. The residue was purified by flash chromatography (100\% EtOAc) to give pure product trans 7a (60 mg, 73\% yield) as a black solid. Black solid, M.p. $78-80{ }^{\circ} \mathrm{C}{ }^{1} \mathrm{H}$ NMR $\left(\mathrm{CDCl}_{3}, 300 \mathrm{MHz}\right): \delta=7.45(\mathrm{~d}, J=8.3 \mathrm{~Hz}, 2 \mathrm{H}), 7.26(\mathrm{~d}, J=8.3 \mathrm{~Hz}, 2 \mathrm{H}), 4.60$ (quint, $J=3.0 \mathrm{~Hz}, 1$ H), $4.15(\mathrm{dd}, J=10.7,3.0 \mathrm{~Hz}, 1 \mathrm{H}), 3.31(\mathrm{td}, J=12.1,3.0 \mathrm{~Hz}, 1 \mathrm{H}), 3.08(\mathrm{bs}, \mathrm{NH}), 3.01(\mathrm{ddd}, J=12.1$, 4.4, $2.6 \mathrm{~Hz}, 1 \mathrm{H}), 2.13-1.91(\mathrm{~m}, 4 \mathrm{H}) .{ }^{13} \mathrm{C} \mathrm{NMR}\left(\mathrm{CDCl}_{3}, 75 \mathrm{MHz}\right): \delta=142.7(\mathrm{C}), 131.7(2 \times \mathrm{CH})$, $128.7(2 \times \mathrm{CH}), 121.3(\mathrm{C}), 57.9(\mathrm{CH}), 54.8(\mathrm{CH}), 42.0\left(\mathrm{CH}_{2}\right), 41.2\left(\mathrm{CH}_{2}\right), 33.2\left(\mathrm{CH}_{2}\right)$. ESI-HRMS calculated for $\mathrm{C}_{11} \mathrm{H}_{14} \mathrm{NClBr}[\mathrm{M}+\mathrm{H}]^{+}$273.9993, found 273.9993 .

\section{General procedure}

To a solution of homoallylic amine (1 equiv., $0.4 \mathrm{mmol})$ and aldehyde (1 equiv*., $0.4 \mathrm{mmol})$ in $\mathrm{CH}_{2} \mathrm{Cl}_{2}(4 \mathrm{~mL})$ p-TSA. $\mathrm{H}_{2} \mathrm{O}$ (0.1 equiv., $\left.0.04 \mathrm{mmol}\right)$ was added. The mixture was stirred for $15 \mathrm{~min}$ 
then a solution of $\mathrm{TiCl}_{4}\left(1 \mathrm{M}\right.$ in $\mathrm{CH}_{2} \mathrm{Cl}_{2}, 1$ equiv., $\left.0.4 \mathrm{mmol}\right)$ was added. The solution was stirred at 60 ${ }^{\circ} \mathrm{C}$ for $16 \mathrm{~h}$ (with amine $\mathbf{1}, \mathbf{4}, \mathbf{1 5}, \mathbf{1 7}$ and 19), $90 \mathrm{~h}$ (with amine $\mathbf{6}^{* *}$ ) or $160 \mathrm{~h}$ (with amine $\mathbf{8}, 10$ and 12), quenched with $\mathrm{NaHCO}_{3}$ and extracted with $\mathrm{CH}_{2} \mathrm{Cl}_{2}$. The combined organic layers were dried with $\mathrm{MgSO}_{4}$, solvent was removed, and the residue was purified by flash chromatography on silica gel (10\% EtOAc in petroleum ether) to give the pure piperidine products.

* 1.5 eq for aldehyde $\mathbf{2 m}$ and $\mathbf{2 n}$

** For amine $\mathbf{6}$ reaction with $\mathbf{2 j}$ and $2 \mathbf{k}$ has been extended for $160 \mathrm{~h}$

Methyl 2-(2-(4-bromophenyl)-4-chloropiperidin-1-yl)acetate 3a (98\%, $136 \mathrm{mg}$, dr: 57:43)

Cis 3a: Orange oil ${ }^{1} \mathrm{H}$ NMR $\left(\mathrm{CDCl}_{3}, 300 \mathrm{MHz}\right): \delta=7.45(\mathrm{~d}, J=8.4 \mathrm{~Hz}, 2 \mathrm{H}), 7.22(\mathrm{~d}, J=8.4 \mathrm{~Hz}, 2$ H), $3.94(\mathrm{tt}, J=11.7,4.3 \mathrm{~Hz}, 1 \mathrm{H}), 3.63(\mathrm{dd}, J=12.1,2.2 \mathrm{~Hz}, 1 \mathrm{H}), 3.61(\mathrm{~s}, 3 \mathrm{H}), 3.08(\mathrm{ddd}, J=12.1$, 4.1, $2.8 \mathrm{~Hz}, 1 \mathrm{H}), 3.07(\mathrm{ABq}, 2 \mathrm{H}), 2.66(\mathrm{td}, J=12.1,2.2 \mathrm{~Hz}, 1 \mathrm{H}), 2.24-2.15(\mathrm{~m}, 2 \mathrm{H}), 2.06$ (qd, $J=$ 12.1, $4.1 \mathrm{~Hz}, 1 \mathrm{H}), 1.89$ (q, $J=12.1 \mathrm{~Hz}, 1 \mathrm{H}) .{ }^{13} \mathrm{C} \mathrm{NMR}\left(\mathrm{CDCl}_{3}, 75 \mathrm{MHz}\right): \delta=171.1(\mathrm{C}), 141.1(\mathrm{C})$, $132.1(2 \times \mathrm{C}), 129.5(2 \times \mathrm{C}), 121.7(\mathrm{C}), 64.9(\mathrm{C}), 56.5(\mathrm{C}), 54.9(\mathrm{C}), 52.4(\mathrm{C}), 51.5(\mathrm{C}), 45.9(\mathrm{C}), 36.8$ (C). ESI-HRMS calculated for $\mathrm{C}_{14} \mathrm{H}_{17} \mathrm{NO}_{2} \mathrm{ClBrNa}[\mathrm{M}+\mathrm{Na}]^{+}$368.0029, found 368.0032. Trans 3a: Yellow oil ${ }^{1} \mathrm{H}$ NMR $\left(\mathrm{CDCl}_{3}, 300 \mathrm{MHz}\right): \delta=7.45(\mathrm{~d}, J=8.5 \mathrm{~Hz}, 2 \mathrm{H}), 7.25(\mathrm{~d}, J=8.5 \mathrm{~Hz}, 2 \mathrm{H}), 4.53$ (quint, $J=3.0 \mathrm{~Hz}, 1 \mathrm{H}), 3.95(\mathrm{dd}, J=10,3.8 \mathrm{~Hz}, 1 \mathrm{H}), 3.64(\mathrm{~s}, 3 \mathrm{H}), 3.11(\mathrm{ABq}, 2 \mathrm{H}), 3.05-2.92(\mathrm{~m}, 2$ $\mathrm{H}-\mathrm{H}), 2.32-2.21(\mathrm{~m}, 1 \mathrm{H}), 2.10-1.95(\mathrm{~m}, 3 \mathrm{H}) .{ }^{13} \mathrm{C} \mathrm{NMR}\left(\mathrm{CDCl}_{3}, 75 \mathrm{MHz}\right): \delta=171.3(\mathrm{C}), 141.6(\mathrm{C})$, $132.1(2 \times \mathrm{C}), 129.7(2 \times \mathrm{C}), 121.5(\mathrm{C}), 60.0(\mathrm{C}), 57.0(\mathrm{C}), 56.0(\mathrm{C}), 51.6(\mathrm{C}), 47.4(\mathrm{C}), 43.1(\mathrm{C}), 33.4$ (C). ESI-HRMS calculated for $\mathrm{C}_{14} \mathrm{H}_{17} \mathrm{NO}_{2} \mathrm{ClBrNa}[\mathrm{M}+\mathrm{Na}]^{+} 368.0028$, found 368.0028 .

Methyl 2-(4-chloro-2-phenylpiperidin-1-yl)acetate $3 \boldsymbol{b}$ (94\%, $100 \mathrm{mg}$, dr: 57:43)

Cis 3b: Yellow oil ${ }^{1} \mathrm{H}$ NMR $\left(\mathrm{CDCl}_{3}, 300 \mathrm{MHz}\right)$ : $\delta=7.33-7.26(\mathrm{~m}, 5 \mathrm{H}), 3.96(\mathrm{tt}, J=11.7,4.3 \mathrm{~Hz}, 1$ H), $3.62(\mathrm{dd}, J=10.9,2.6 \mathrm{~Hz}, 1 \mathrm{H}), 3.60(\mathrm{~s}, 3 \mathrm{H}), 3.10(\mathrm{ddd}, J=11.9,4.1,2.9 \mathrm{~Hz}, 1 \mathrm{H}), 3.08(\mathrm{ABq}, 2$ H), $2.66(\mathrm{td}, J=12.0,2.6 \mathrm{~Hz}, 1 \mathrm{H}), 2.21(\mathrm{~m}, 2 \mathrm{H}), 2.07(\mathrm{qd}, J=12.0,4.2 \mathrm{~Hz}, 1 \mathrm{H}), 1.96(\mathrm{q}, J=12.0$ $\mathrm{Hz}, 1 \mathrm{H}) .{ }^{13} \mathrm{C} \mathrm{NMR}\left(\mathrm{CDCl}_{3}, 75 \mathrm{MHz}\right): \delta=171.3(\mathrm{C}), 142.0(\mathrm{C}), 128.9(2 \times \mathrm{CH}), 128.0(\mathrm{CH}), 127.7(2$ $\times \mathrm{CH}), 65.7(\mathrm{CH}), 56.9(\mathrm{CH}), 55.0\left(\mathrm{CH}_{2}\right), 52.5\left(\mathrm{CH}_{2}\right), 51.4\left(\mathrm{CH}_{3}\right), 46.0\left(\mathrm{CH}_{2}\right), 36.9\left(\mathrm{CH}_{2}\right)$. ESI-HRMS calculated for $\mathrm{C}_{14} \mathrm{H}_{18} \mathrm{NO}_{2} \mathrm{ClNa}[\mathrm{M}+\mathrm{Na}]^{+} 290.0924$, found 290.0922. Trans 3b: Yellow oil ${ }^{1} \mathrm{H}$ NMR $\left(\mathrm{CDCl}_{3}, 300 \mathrm{MHz}\right): \delta=7.38-7.25(\mathrm{~m}, 5 \mathrm{H}), 4.54$ (quint, $\left.J=2.9 \mathrm{~Hz}, 1 \mathrm{H}\right), 3.94(\mathrm{dd}, J=10.8,3.0 \mathrm{~Hz}, 1$ 
$\mathrm{H}), 3.63(\mathrm{~s}, 3 \mathrm{H}), 3.11(\mathrm{ABq}, 2 \mathrm{H}), 3.01-2.91(\mathrm{~m}, 2 \mathrm{H}), 2.30-2.28(\mathrm{~m}, 1 \mathrm{H}), 2.17-1.96(\mathrm{~m}, 3 \mathrm{H}) .{ }^{13} \mathrm{C}$ $\mathrm{NMR}\left(\mathrm{CDCl}_{3}, 75 \mathrm{MHz}\right): \delta=171.4(\mathrm{C}), 142.4(\mathrm{C}), 128.9(2 \times \mathrm{CH}), 127.9(2 \times \mathrm{CH}), 127.8(\mathrm{CH}), 60.7$ $(\mathrm{CH}), 57.3(\mathrm{CH}), 56.1\left(\mathrm{CH}_{2}\right), 51.5\left(\mathrm{CH}_{3}\right), 47.5\left(\mathrm{CH}_{2}\right), 43.1\left(\mathrm{CH}_{2}\right), 33.5\left(\mathrm{CH}_{2}\right)$. ESI-HRMS calculated for $\mathrm{C}_{14} \mathrm{H}_{18} \mathrm{NO}_{2} \mathrm{ClNa}[\mathrm{M}+\mathrm{Na}]^{+} 290.0924$, found 290.0926 .

Methyl 2-(4-chloro-2-(2-chlorophenyl)piperidin-1-yl)acetate 3c (86\%, $103 \mathrm{mg}$, dr: 86:14)

Cis 3c: Orange oil ${ }^{1} \mathrm{H}$ NMR $\left(\mathrm{CDCl}_{3}, 300 \mathrm{MHz}\right): \delta=7.61(\mathrm{dd}, J=7.6,1.7 \mathrm{~Hz}, 1 \mathrm{H}), 7.33(\mathrm{dd}, J=7.9$, $1.3 \mathrm{~Hz}, 1 \mathrm{H}), 7.28(\mathrm{td}, J=7.6,1.3 \mathrm{~Hz}, 1 \mathrm{H}), 7.18(\mathrm{td}, J=7.9,1.7 \mathrm{~Hz}, 1 \mathrm{H}), 4.10(\mathrm{dd}, J=11.2,3.1 \mathrm{~Hz}$, $1 \mathrm{H}), 3.97(\mathrm{tt}, J=11.7,4.3 \mathrm{~Hz}, 1 \mathrm{H}), 3.63(\mathrm{~s}, 3 \mathrm{H}), 3.18(\mathrm{dt}, J=11.8,3.5 \mathrm{~Hz}, 1 \mathrm{H}), 3.08(\mathrm{ABq}, 2 \mathrm{H})$, $2.60(\mathrm{td}, J=12.0,2.5 \mathrm{~Hz}, 1 \mathrm{H}), 2.32-2.19(\mathrm{~m}, 2 \mathrm{H}), 2.06(\mathrm{qd}, J=12.1,4.1 \mathrm{~Hz}, 1 \mathrm{H}), 1.79(\mathrm{q}, J=12.1$ $\mathrm{Hz}, 1 \mathrm{H}) .{ }^{13} \mathrm{C} \mathrm{NMR}\left(\mathrm{CDCl}_{3}, 75 \mathrm{MHz}\right): \delta=171.1(\mathrm{C}), 139.2(\mathrm{C}), 133.2(\mathrm{C}), 129.8(\mathrm{CH}), 128.8(\mathrm{CH})$, 128.6 (CH), $127.7(\mathrm{CH}), 61.2(\mathrm{CH}), 56.4(\mathrm{CH}), 55.3\left(\mathrm{CH}_{2}\right), 52.8\left(\mathrm{CH}_{2}\right), 51.6\left(\mathrm{CH}_{3}\right), 44.5\left(\mathrm{CH}_{2}\right), 36.7$ $\left(\mathrm{CH}_{2}\right)$. ESI-HRMS calculated for $\mathrm{C}_{14} \mathrm{H}_{17} \mathrm{NO}_{2} \mathrm{Cl}_{2} \mathrm{Na}[\mathrm{M}+\mathrm{Na}]^{+}$324.0534, found 324.0536. Trans 3c: Yellow oil ${ }^{1} \mathrm{H} \mathrm{NMR}\left(\mathrm{CDCl}_{3}, 300 \mathrm{MHz}\right): \delta=7.62(\mathrm{dd}, J=7.6,1.7 \mathrm{~Hz}, 1 \mathrm{H}), 7.33(\mathrm{dd}, J=7.9,1.3 \mathrm{~Hz}$, $1 \mathrm{H}), 7.26(\mathrm{td}, J=7.6,1.3 \mathrm{~Hz}, 1 \mathrm{H}), 7.17(\mathrm{td}, J=7.9,1.7 \mathrm{~Hz}, 1 \mathrm{H}), 4.53$ (quint, $J=4.5 \mathrm{~Hz}, 1 \mathrm{H}$ ), 4.46 (dd, $J=11.0,2.9 \mathrm{~Hz}, 1 \mathrm{H}), 3.66(\mathrm{~s}, 3 \mathrm{H}), 3.09(\mathrm{ABq}, 2 \mathrm{H}), 3.01-2.96(\mathrm{~m}, 2 \mathrm{H}), 2.29-2.22(\mathrm{~m}, 1 \mathrm{H})$, 2.13-2.08 (m, $1 \mathrm{H}), 2.02-1.87$ (m, $2 \mathrm{H}) .{ }^{13} \mathrm{C} \mathrm{NMR}\left(\mathrm{CDCl}_{3}, 75 \mathrm{MHz}\right): \delta=171.4(\mathrm{C}), 139.9(\mathrm{C}), 133.6$ (C), $129.9(\mathrm{CH}), 128.9(\mathrm{CH}), 128.5(\mathrm{CH}), 127.6(\mathrm{CH}), 56.8(\mathrm{CH}), 56.4(\mathrm{CH}), 56.4\left(\mathrm{CH}_{2}\right), 51.7\left(\mathrm{CH}_{3}\right)$, $47.7\left(\mathrm{CH}_{2}\right), 41.7\left(\mathrm{CH}_{2}\right), 33.4\left(\mathrm{CH}_{2}\right)$. ESI-HRMS calculated for $\mathrm{C}_{14} \mathrm{H}_{17} \mathrm{NO}_{2} \mathrm{Cl}_{2} \mathrm{Na}[\mathrm{M}+\mathrm{Na}]^{+} 324.0534$, found 324.0535 .

Methyl 2-(4-chloro-2-(4-cyanophenyl)piperidin-1-yl)acetate 3d (96\%, $112 \mathrm{mg}$, dr: 42:58)

Cis 3d: Orange oil ${ }^{1} \mathrm{H}$ NMR $\left(\mathrm{CDCl}_{3}, 300 \mathrm{MHz}\right): \delta=7.64$ (d, $\left.J=8.3 \mathrm{~Hz}, 2 \mathrm{H}\right), 7.47(\mathrm{~d}, J=8.3 \mathrm{~Hz}, 2$ H), $3.95(\mathrm{tt}, J=11.7,4.2 \mathrm{~Hz}, 1 \mathrm{H}), 3.78(\mathrm{dd}, J=12.1,2.4 \mathrm{~Hz}, 1 \mathrm{H}), 3.62(\mathrm{~s}, 3 \mathrm{H}), 3.10(\mathrm{ddd}, J=11.9$, 3.9, $2.9 \mathrm{~Hz}, 1 \mathrm{H}), 3.06(\mathrm{ABq}, 2 \mathrm{H}), 2.70(\mathrm{td}, J=12.1,2.3 \mathrm{~Hz}, 1 \mathrm{H}), 2.26-2.16(\mathrm{~m}, 2 \mathrm{H}), 2.04(\mathrm{qd}, J=$ 12.1, $4.2 \mathrm{~Hz}, 1 \mathrm{H}), 1.86$ (q, $J=11.7 \mathrm{~Hz}, 1 \mathrm{H}) .{ }^{13} \mathrm{C} \mathrm{NMR}\left(\mathrm{CDCl}_{3}, 75 \mathrm{MHz}\right): \delta=170.8(\mathrm{C}), 147.6(\mathrm{C})$, $132.8(2 \times \mathrm{CH}), 128.5(2 \times \mathrm{CH}), 118.7(\mathrm{C}), 111.8(\mathrm{C}), 65.0(\mathrm{CH}), 56.2(\mathrm{CH}), 54.8\left(\mathrm{CH}_{2}\right), 52.2\left(\mathrm{CH}_{2}\right)$, $51.5\left(\mathrm{CH}_{3}\right), 45.7\left(\mathrm{CH}_{2}\right), 36.6\left(\mathrm{CH}_{2}\right)$. ESI-HRMS calculated for $\mathrm{C}_{15} \mathrm{H}_{17} \mathrm{~N}_{2} \mathrm{O}_{2} \mathrm{ClNa}[\mathrm{M}+\mathrm{Na}]^{+}$315.0876, found 315.0877. Trans 3d: Orange oil ${ }^{1} \mathrm{H} \mathrm{NMR}\left(\mathrm{CDCl}_{3}, 300 \mathrm{MHz}\right): \delta=7.64(\mathrm{~d}, J=8.6 \mathrm{~Hz}, 2 \mathrm{H}), 7.51$ 
$(\mathrm{d}, J=8.6 \mathrm{~Hz}, 2 \mathrm{H}), 4.53$ (quint, $J=2.9 \mathrm{~Hz}, 1 \mathrm{H}), 4.07(\mathrm{t}, J=6.8 \mathrm{~Hz}, 1 \mathrm{H}), 3.65(\mathrm{~s}, 3 \mathrm{H}), 3.07(\mathrm{ABq}, 2$ $\mathrm{H}), 3.02(\mathrm{td}, J=11.6,3.0 \mathrm{~Hz}, 1 \mathrm{H}), 2.98-2.93(\mathrm{~m}, 1 \mathrm{H}), 2.27-2.22(\mathrm{~m}, 1 \mathrm{H}), 2.02-1.98(\mathrm{~m}, 3 \mathrm{H}) .{ }^{13} \mathrm{C}$ $\mathrm{NMR}\left(\mathrm{CDCl}_{3}, 75 \mathrm{MHz}\right): \delta=171.0(\mathrm{C}), 148.3(\mathrm{C}), 132.7(2 \times \mathrm{CH}), 128.6(2 \times \mathrm{CH}), 117.7(\mathrm{C}), 111.6$ (C), $60.1(\mathrm{CH}), 56.5(\mathrm{CH}), 56.0\left(\mathrm{CH}_{2}\right), 51.6\left(\mathrm{CH}_{3}\right), 47.1\left(\mathrm{CH}_{2}\right), 43.0\left(\mathrm{CH}_{2}\right), 33.2\left(\mathrm{CH}_{2}\right)$. ESI-HRMS calculated for $\mathrm{C}_{15} \mathrm{H}_{17} \mathrm{~N}_{2} \mathrm{O}_{2} \mathrm{ClNa}[\mathrm{M}+\mathrm{Na}]^{+}$315.0876, found 315.0876.

Methyl 2-(4-chloro-2-(4-nitrophenyl)piperidin-1-yl)acetate 3e (90\%, $111 \mathrm{mg}, \mathrm{dr}$ : 38:62)

Cis 3e: Orange oil ${ }^{1} \mathrm{H}$ NMR $\left(\mathrm{CDCl}_{3}, 300 \mathrm{MHz}\right): \delta=8.20(\mathrm{~d}, J=8.8 \mathrm{~Hz}, 2 \mathrm{H}), 7.53(\mathrm{~d}, J=8.6 \mathrm{~Hz}, 2$ H), $3.96(\mathrm{tt}, J=11.7,4.3 \mathrm{~Hz}, 1 \mathrm{H}), 3.86(\mathrm{dd}, J=11.4,2.5 \mathrm{~Hz}, 1 \mathrm{H}), 3.62(\mathrm{~s}, 3 \mathrm{H}), 3.11$ (ddd, $J=11.9$, 4.1, 3.0 Hz, $1 \mathrm{H}), 3.07(\mathrm{ABq}, 2 \mathrm{H}), 2.72(\mathrm{td}, J=12.2,2.5 \mathrm{~Hz}, 1 \mathrm{H}), 2.28-2.17(\mathrm{~m}, 2 \mathrm{H}), 2.07$ (qd, $J=$ 12.2, $4.1 \mathrm{~Hz}, 1 \mathrm{H}), 1.88$ (q, $J=11.9 \mathrm{~Hz}, 1 \mathrm{H}) .{ }^{13} \mathrm{C} \mathrm{NMR}\left(\mathrm{CDCl}_{3}, 75 \mathrm{MHz}\right): \delta=170.8(\mathrm{C}), 149.6(\mathrm{C})$, $147.7(\mathrm{C}), 128.6(2 \times \mathrm{CH}), 124.3(2 \times \mathrm{CH}), 64.7(\mathrm{CH}), 56.1(\mathrm{CH}), 54.8\left(\mathrm{CH}_{2}\right), 52.2\left(\mathrm{CH}_{2}\right), 51.6\left(\mathrm{CH}_{3}\right)$, $45.8\left(\mathrm{CH}_{2}\right), 36.7\left(\mathrm{CH}_{2}\right)$. ESI-HRMS calculated for $\mathrm{C}_{14} \mathrm{H}_{17} \mathrm{~N}_{2} \mathrm{O}_{4} \mathrm{ClNa}[\mathrm{M}+\mathrm{Na}]^{+}$335.0775, found 335.0775. Trans 3e: Orange solid M.p. $112-114{ }^{\circ} \mathrm{C} .{ }^{1} \mathrm{H}$ NMR $\left(\mathrm{CDCl}_{3}, 300 \mathrm{MHz}\right): \delta=8.20(\mathrm{~d}, J=8.8$ $\mathrm{Hz}, 2 \mathrm{H}), 7.59$ (d, $J=8.6 \mathrm{~Hz}, 2 \mathrm{H}), 4.54$ (quint, $J=3.0 \mathrm{~Hz}, 1 \mathrm{H}), 4.15$ (t, $J=7.0 \mathrm{~Hz}, 1 \mathrm{H}), 3.65$ (s, 3 $\mathrm{H}), 3.08(\mathrm{ABq}, 2 \mathrm{H}), 3.05-2.94(\mathrm{~m}, 2 \mathrm{H}), 2.28-2.22(\mathrm{~m}, 1 \mathrm{H}), 2.04-2.00(\mathrm{~m}, 3 \mathrm{H}) .{ }^{13} \mathrm{C} \mathrm{NMR}\left(\mathrm{CDCl}_{3}, 75\right.$ MHz): $\delta=171.0(\mathrm{C}), 150.4(\mathrm{C}), 147.6(\mathrm{C}), 128.7(2 \times \mathrm{CH}), 124.2(2 \times \mathrm{CH}), 59.9(\mathrm{CH}), 56.5(\mathrm{CH})$, 56.0 $\left(\mathrm{CH}_{2}\right), \quad 51.6\left(\mathrm{CH}_{3}\right), 47.1\left(\mathrm{CH}_{2}\right), 43.1 \quad\left(\mathrm{CH}_{2}\right), 33.2\left(\mathrm{CH}_{2}\right)$. ESI-HRMS calculated for $\mathrm{C}_{14} \mathrm{H}_{17} \mathrm{~N}_{2} \mathrm{O}_{4} \mathrm{ClNa}[\mathrm{M}+\mathrm{Na}]^{+}$335.0775, found 335.0775.

Methyl 2-(4-chloro-2-(3-nitrophenyl)piperidin-1-yl)acetate $3 f$ (97\%, $122 \mathrm{mg}$, dr: 45:55)

Cis 3f: Yellow solid M.p. $98-100{ }^{\circ} \mathrm{C} .{ }^{1} \mathrm{H}$ NMR $\left(\mathrm{CDCl}_{3}, 300 \mathrm{MHz}\right): \delta=8.21$ (s, $\left.1 \mathrm{H}\right), 8.15$ (d, $J=8.1$ $\mathrm{Hz}, 1 \mathrm{H}), 7.71(\mathrm{~d}, J=7.6 \mathrm{~Hz}, 1 \mathrm{H}), 7.53(\mathrm{t}, J=7.9 \mathrm{~Hz}, 1 \mathrm{H}), 3.97(\mathrm{tt}, J=11.7,4.3 \mathrm{~Hz}, 1 \mathrm{H}), 3.86$ (dd, $J=11.4,2.3 \mathrm{~Hz}, 1 \mathrm{H}), 3.62(\mathrm{~s}, 3 \mathrm{H}), 3.11(\mathrm{ddd}, J=11.7,3.9,2.6 \mathrm{~Hz}, 1 \mathrm{H}), 3.09(\mathrm{ABq}, 2 \mathrm{H}), 2.72(\mathrm{td}, J$ $=12.2,2.3 \mathrm{~Hz}, 1 \mathrm{H}), 2.26-2.22(\mathrm{~m}, 2 \mathrm{H}), 2.06(\mathrm{qd}, J=12.2,4.1 \mathrm{~Hz}, 1 \mathrm{H}), 1.90(\mathrm{q}, J=11.9 \mathrm{~Hz}, 1 \mathrm{H})$.

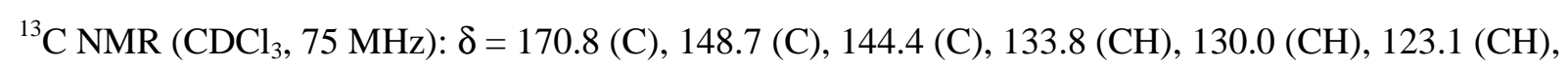
$122.8(\mathrm{CH}), 64.5(\mathrm{CH}), 56.1(\mathrm{CH}), 54.8\left(\mathrm{CH}_{2}\right), 52.2\left(\mathrm{CH}_{2}\right), 51.5\left(\mathrm{CH}_{3}\right), 46.0\left(\mathrm{CH}_{2}\right), 36.7\left(\mathrm{CH}_{2}\right)$. ESIHRMS calculated for $\mathrm{C}_{14} \mathrm{H}_{17} \mathrm{~N}_{2} \mathrm{O}_{4} \mathrm{ClNa}[\mathrm{M}+\mathrm{Na}]^{+}$335.0775, found 335.0777. Trans 3f: Orange oil ${ }^{1} \mathrm{H}$ $\operatorname{NMR}\left(\mathrm{CDCl}_{3}, 300 \mathrm{MHz}\right): \delta=8.26(\mathrm{t}, J=1.1 \mathrm{~Hz}, 1 \mathrm{H}), 8.14(\mathrm{qd}, J=8.1,1.1 \mathrm{~Hz}, 1 \mathrm{H}), 7.75(\mathrm{~d}, J=7.7$ 
$\mathrm{Hz}, 1 \mathrm{H}), 7.52(\mathrm{~d}, J=7.9 \mathrm{~Hz}, 1 \mathrm{H}), 4.55$ (quint, $J=2.9 \mathrm{~Hz}, 1 \mathrm{H}), 4.17$ (t, $J=7.0 \mathrm{~Hz}, 1 \mathrm{H}), 3.65(\mathrm{~s}, 3$ $\mathrm{H}), 3.09(\mathrm{dt}, J=11.9,2.5 \mathrm{~Hz}, 1 \mathrm{H}), 3.08(\mathrm{ABq}, 2 \mathrm{H}), 2.98-2.92(\mathrm{~m}, 1 \mathrm{H}), 2.33-2.23(\mathrm{~m}, 1 \mathrm{H}), 2.07-2.03$ $(\mathrm{m}, 3 \mathrm{H}) .{ }^{13} \mathrm{C} \mathrm{NMR}\left(\mathrm{CDCl}_{3}, 75 \mathrm{MHz}\right): \delta=170.9(\mathrm{C}), 148.7(\mathrm{C}), 145.0(\mathrm{C}), 134.1(\mathrm{CH}), 129.9(\mathrm{CH})$, $129.9(\mathrm{CH}), 122.9(\mathrm{CH}), 59.7(\mathrm{CH}), 56.6(\mathrm{CH}), 56.0\left(\mathrm{CH}_{2}\right), 51.6\left(\mathrm{CH}_{3}\right), 47.1\left(\mathrm{CH}_{2}\right), 43.2\left(\mathrm{CH}_{2}\right), 33.3$ $\left(\mathrm{CH}_{2}\right)$. ESI-HRMS calculated for $\mathrm{C}_{14} \mathrm{H}_{17} \mathrm{~N}_{2} \mathrm{O}_{4} \mathrm{ClNa}[\mathrm{M}+\mathrm{Na}]^{+}$335.0775, found 335.0775.

Methyl 2-(4-chloro-2-(4-(dimethylamino)phenyl)piperidin-1-yl)acetate $3 g$ (60\%, $75 \mathrm{mg}$, dr: 50:50)

Cis 3g: White solid M.p. $88-90{ }^{\circ} \mathrm{C} .{ }^{1} \mathrm{H}$ NMR $\left(\mathrm{CDCl}_{3}, 300 \mathrm{MHz}\right): \delta=7.17$ (d, $\left.J=8.7 \mathrm{~Hz}, 2 \mathrm{H}\right), 6.68$ $(\mathrm{d}, J=8.7 \mathrm{~Hz}, 2 \mathrm{H}), 3.95(\mathrm{tt}, J=11.7,4.4 \mathrm{~Hz}, 1 \mathrm{H}), 3.60(\mathrm{~s}, 3 \mathrm{H}), 3.48(\mathrm{dd}, J=11.4,2.4 \mathrm{~Hz}, 1 \mathrm{H})$, $3.10(\mathrm{ABq}, 2 \mathrm{H}), 3.09$ (dt, $J=11.8,3.4 \mathrm{~Hz}, 1 \mathrm{H}), 2.94(\mathrm{~s}, 6 \mathrm{H}), 2.61(\mathrm{td}, J=12.0,2.4 \mathrm{~Hz}, 1 \mathrm{H}), 2.22-$ 2.17 (m, $2 \mathrm{H}), 2.12-1.93(\mathrm{~m}, 2 \mathrm{H}) .{ }^{13} \mathrm{C} \mathrm{NMR}\left(\mathrm{CDCl}_{3}, 75 \mathrm{MHz}\right): \delta=171.5(\mathrm{C}), 150.3(\mathrm{C}), 129.5(\mathrm{C})$, $128.5(2 \times \mathrm{CH}), 112.7(2 \times \mathrm{CH}), 65.2(\mathrm{CH}), 57.2(\mathrm{CH}), 54.9\left(\mathrm{CH}_{2}\right), 52.5\left(\mathrm{CH}_{2}\right), 51.3\left(\mathrm{CH}_{3}\right), 45.8$ $\left(\mathrm{CH}_{2}\right), 40.7\left(2 \times \mathrm{CH}_{3}\right), 36.9\left(\mathrm{CH}_{2}\right)$. ESI-HRMS calculated for $\mathrm{C}_{16} \mathrm{H}_{23} \mathrm{~N}_{2} \mathrm{O}_{2} \mathrm{ClNa}[\mathrm{M}+\mathrm{Na}]^{+} 333.1346$, found 333.1345. Trans 3g: Brown solid M.p. $60-62{ }^{\circ} \mathrm{C} .{ }^{1} \mathrm{H}$ NMR $\left(\mathrm{CDCl}_{3}, 300 \mathrm{MHz}\right): \delta=7.20(\mathrm{~d}, J=$ $8.8 \mathrm{~Hz}, 2 \mathrm{H}), 6.68(\mathrm{~d}, J=8.8 \mathrm{~Hz}, 2 \mathrm{H}), 4.54$ (quint, $J=2.9 \mathrm{~Hz}, 1 \mathrm{H}), 3.80(\mathrm{dd}, J=10.9,2.8 \mathrm{~Hz}, 1 \mathrm{H})$, 3.62 (s, 3 H), 3.11 (ABq, 2 H), 2.94-2.89 (m, 8 H), 2.32-2.22 (m, 1 H), 2.17-2.08 (m, 1 H), 2.02-1.93 (m, $2 \mathrm{H}) .{ }^{13} \mathrm{C} \mathrm{NMR}\left(\mathrm{CDCl}_{3}, 75 \mathrm{MHz}\right): \delta=171.7(\mathrm{C}), 150.2(\mathrm{C}), 129.9(\mathrm{C}), 128.7(2 \times \mathrm{CH}), 112.7(2 \times$ $\mathrm{CH}), 60.0(\mathrm{CH}), 57.8(\mathrm{CH}), 56.1\left(\mathrm{CH}_{2}\right), 51.4\left(\mathrm{CH}_{3}\right), 47.6\left(\mathrm{CH}_{2}\right), 42.9\left(\mathrm{CH}_{2}\right), 40.7\left(2 \times \mathrm{CH}_{3}\right), 33.6$ $\left(\mathrm{CH}_{2}\right)$. ESI-HRMS calculated for $\mathrm{C}_{16} \mathrm{H}_{23} \mathrm{~N}_{2} \mathrm{O}_{2} \mathrm{ClNa}[\mathrm{M}+\mathrm{Na}]^{+}$333.1346, found 333.1348.

Methyl 2-(4-chloro-2-(2,5-dimethoxyphenyl)piperidin-1-yl)acetate $3 \boldsymbol{h}$ (92\%, $120 \mathrm{mg}$, dr: 87:13)

Cis 3h: Yellow solid M.p. $78-80{ }^{\circ} \mathrm{C} .{ }^{1} \mathrm{H}$ NMR $\left(\mathrm{CDCl}_{3}, 300 \mathrm{MHz}\right): \delta=7.10(\mathrm{~d}, J=2.85 \mathrm{~Hz}, 1 \mathrm{H})$, 6.81-6.73 (m, 2 H), 3.98-3.92 (m, 2 H), 3.77 (s, 3 H), 3.76 (s, 3 H), 3.62 (s, 3 H), 3.16 (dt, $J=11.7,3.5$ $\mathrm{Hz}, 1 \mathrm{H}), 3.07(\mathrm{ABq}, 2 \mathrm{H}), 2.52(\mathrm{td}, J=12.0,2.0 \mathrm{~Hz}, 1 \mathrm{H}), 2.26-2.17(\mathrm{~m}, 2 \mathrm{H}), 2.08(\mathrm{qd}, J=12.0,4.0$ $\mathrm{Hz}, 1 \mathrm{H}), 1.87$ (q, $J=12.0 \mathrm{~Hz}, 1 \mathrm{H}) .{ }^{13} \mathrm{C} \mathrm{NMR}\left(\mathrm{CDCl}_{3}, 75 \mathrm{MHz}\right): \delta=171.4(\mathrm{C}), 154.3(\mathrm{C}), 151.0(\mathrm{C})$, $130.9(\mathrm{C}), 113.9(\mathrm{CH}), 112.9(\mathrm{CH}), 112.2(\mathrm{CH}), 58.1(\mathrm{CH}), 56.9(\mathrm{CH}), 56.1\left(\mathrm{CH}_{3}\right), 55.8\left(\mathrm{CH}_{3}\right), 55.6$ $\left(\mathrm{CH}_{2}\right), 53.2\left(\mathrm{CH}_{2}\right), 51.5\left(\mathrm{CH}_{3}\right), 44.6(\mathrm{CH}), 36.7(\mathrm{CH})$. ESI-HRMS calculated for $\mathrm{C}_{16} \mathrm{H}_{22} \mathrm{NO}_{4} \mathrm{ClNa}$ $[\mathrm{M}+\mathrm{Na}]^{+}$350.1135, found 350.1139. Trans 3h: Yellow solid M.p. $72-74{ }^{\circ} \mathrm{C} .{ }^{1} \mathrm{H}$ NMR $\left(\mathrm{CDCl}_{3}, 300\right.$ MHz): $\delta=7.11(\mathrm{~d}, J=3.00 \mathrm{~Hz}, 1 \mathrm{H}), 6.81-6.75(\mathrm{~m}, 2 \mathrm{H}), 4.53$ (quint, $J=2.9 \mathrm{~Hz}, 1 \mathrm{H}), 4.32(\mathrm{dd}, J=$ 
7.7, $5.9 \mathrm{~Hz}, 1 \mathrm{H}), 3.78$ (s, $3 \mathrm{H}), 3.76$ (s, $3 \mathrm{H}), 3.64$ (s, $3 \mathrm{H}), 3.10(\mathrm{ABq}, 2 \mathrm{H}), 2.99-2.86$ (m, $2 \mathrm{H}), 2.29-$ $2.24(\mathrm{~m}, 1 \mathrm{H}), 2.03-2.01(\mathrm{~m}, 3 \mathrm{H}) .{ }^{13} \mathrm{C} \mathrm{NMR}\left(\mathrm{CDCl}_{3}, 75 \mathrm{MHz}\right): \delta=171.8(\mathrm{C}), 154.3(\mathrm{C}), 151.5(\mathrm{C})$, $131.6(\mathrm{C}), 113.7(\mathrm{CH}), 113.1(\mathrm{CH}), 112.5(\mathrm{CH}), 57.5(\mathrm{CH}), 56.6\left(\mathrm{CH}_{2}\right), 56.5(\mathrm{CH}), 55.9\left(\mathrm{CH}_{3}\right), 53.0$ $\left(\mathrm{CH}_{3}\right), 51.6\left(\mathrm{CH}_{3}\right), 48.1\left(\mathrm{CH}_{2}\right), 41.7\left(\mathrm{CH}_{2}\right), 33.5\left(\mathrm{CH}_{2}\right)$. ESI-HRMS calculated for $\mathrm{C}_{16} \mathrm{H}_{22} \mathrm{NO}_{4} \mathrm{ClNa}$ $[\mathrm{M}+\mathrm{Na}]^{+} 350.1135$, found 350.1144 .

Methyl 2-(4-chloro-2-(2-methoxyphenyl)piperidin-1-yl)acetate $3 \boldsymbol{i}$ (65\%, $78 \mathrm{mg}$, dr: 85:15)

Cis 3i: Orange oil ${ }^{1} \mathrm{H}$ NMR $\left(\mathrm{CDCl}_{3}, 300 \mathrm{MHz}\right): \delta=7.50(\mathrm{dd}, J=7.5,1.7 \mathrm{~Hz}, 1 \mathrm{H}), 7.22(\mathrm{ddd}, J=8.2$, 7.5, 1.7 Hz, $1 \mathrm{H}), 6.97(\mathrm{td}, J=8.2,1.0 \mathrm{~Hz}, 1 \mathrm{H}), 6.85(\mathrm{dd}, J=8.2,1.0 \mathrm{~Hz}, 1 \mathrm{H}), 4.00-3.95(\mathrm{~m}, 2 \mathrm{H})$, $3.80(\mathrm{~s}, 3 \mathrm{H}), 3.61(\mathrm{~s}, 3 \mathrm{H}), 3.18(\mathrm{dt}, J=11.8,3.4 \mathrm{~Hz}, 1 \mathrm{H}), 3.06(\mathrm{Abq}, 2 \mathrm{H}), 2.52(\mathrm{td}, J=12.0,2.5 \mathrm{~Hz}$, $1 \mathrm{H}), 2.23-2.13(\mathrm{~m}, 2 \mathrm{H}), 2.08(\mathrm{qd}, J=12.1,4.0 \mathrm{~Hz}, 1 \mathrm{H}), 1.89(\mathrm{q}, J=12.1 \mathrm{~Hz}, 1 \mathrm{H}) .{ }^{13} \mathrm{C} \mathrm{NMR}$ $\left(\mathrm{CDCl}_{3}, 75 \mathrm{MHz}\right): \delta=171.5(\mathrm{C}), 156.8(\mathrm{C}), 129.8(\mathrm{C}), 128.4(\mathrm{CH}), 128.0(\mathrm{CH}), 121.2(\mathrm{CH}), 110.7$ $(\mathrm{CH}), 57.9(\mathrm{CH}), 57.0(\mathrm{CH}), 55.6\left(\mathrm{CH}_{2}\right), 55.5\left(\mathrm{CH}_{3}\right), 53.2\left(\mathrm{CH}_{2}\right), 51.5\left(\mathrm{CH}_{3}\right), 44.6\left(\mathrm{CH}_{2}\right), 36.8\left(\mathrm{CH}_{2}\right)$. ESI-HRMS calculated for $\mathrm{C}_{15} \mathrm{H}_{21} \mathrm{NO}_{3} \mathrm{Cl}[\mathrm{M}+\mathrm{H}]^{+} 298.1210$, found 298.1211. Trans 3i: Orange oil ${ }^{1} \mathrm{H}$ $\operatorname{NMR}\left(\mathrm{CDCl}_{3}, 300 \mathrm{MHz}\right): \delta=7.50(\mathrm{dd}, J=7.5,1.7 \mathrm{~Hz}, 1 \mathrm{H}), 7.22(\mathrm{ddd}, J=8.2,7.5,1.7 \mathrm{~Hz}, 1 \mathrm{H})$, $6.95(\mathrm{td}, J=8.2,1.0 \mathrm{~Hz}, 1 \mathrm{H}), 6.86(\mathrm{dd}, J=8.2,1.0 \mathrm{~Hz}, 1 \mathrm{H}), 4.54$ (quint, $J=2.8 \mathrm{~Hz}, 1 \mathrm{H}), 4.35(\mathrm{~m}, 1$ H), $3.82(\mathrm{~s}, 3 \mathrm{H}), 3.63$ (s, $3 \mathrm{H}), 3.09$ (ABq, $2 \mathrm{H}), 3.02-2.86(\mathrm{~m}, 2 \mathrm{H}), 2.34-2.26$ (m, $1 \mathrm{H}), 2.06-1.95$ (m, $3 \mathrm{H}) .{ }^{13} \mathrm{C} \mathrm{NMR}\left(\mathrm{CDCl}_{3}, 75 \mathrm{MHz}\right): \delta=171.7(\mathrm{C}), 157.3(\mathrm{C}), 130.3(\mathrm{C}), 128.3(\mathrm{CH}), 128.3(\mathrm{CH}), 121.2$ $(\mathrm{CH}), 110.8(\mathrm{CH}), 57.5(\mathrm{CH}), 56.5\left(\mathrm{CH}_{2}\right), 55.7\left(\mathrm{CH}_{3}\right), 52.8(\mathrm{CH}), 51.6\left(\mathrm{CH}_{3}\right), 48.2\left(\mathrm{CH}_{2}\right), 41.5\left(\mathrm{CH}_{2}\right)$, 33.5 $\left(\mathrm{CH}_{2}\right)$. ESI-HRMS calculated for $\mathrm{C}_{15} \mathrm{H}_{21} \mathrm{NO}_{3} \mathrm{Cl}[\mathrm{M}+\mathrm{H}]^{+} 298.1210$, found 298.1209 .

Methyl 2-(4-chloro-2-(4-methoxyphenyl)piperidin-1-yl)acetate 3j (60\%, $71 \mathrm{mg}$, dr: 62:38)

Cis 3j: Orange oil ${ }^{1} \mathrm{H}$ NMR $\left(\mathrm{CDCl}_{3}, 300 \mathrm{MHz}\right): \delta=7.23(\mathrm{~d}, J=8.6 \mathrm{~Hz}, 2 \mathrm{H}), 6.85(\mathrm{~d}, J=8.6 \mathrm{~Hz}, 2$ H), $3.95(\mathrm{tt}, J=11.8,4.3 \mathrm{~Hz}, 1 \mathrm{H}), 3.80(\mathrm{~s}, 3 \mathrm{H}), 3.60(\mathrm{~s}, 3 \mathrm{H}), 3.56(\mathrm{dd}, J=11.3,2.3 \mathrm{~Hz}, 1 \mathrm{H}), 3.09$ (dt, $J=11.7,3.4 \mathrm{~Hz}, 1 \mathrm{H}), 3.08(\mathrm{ABq}, 2 \mathrm{H}), 2.67(\mathrm{td}, J=12.1,2.4 \mathrm{~Hz}, 1 \mathrm{H}), 2.22-2.17(\mathrm{~m}, 2 \mathrm{H}), 2.05$ $(\mathrm{qd}, J=12.1,4.2 \mathrm{~Hz}, 1 \mathrm{H}), 1.95(\mathrm{q}, J=12.1 \mathrm{~Hz}, 1 \mathrm{H}) .{ }^{13} \mathrm{C} \mathrm{NMR}\left(\mathrm{CDCl}_{3}, 75 \mathrm{MHz}\right): \delta=171.4(\mathrm{C})$, $159.3(\mathrm{C}), 134.0(\mathrm{C}), 128.8(2 \times \mathrm{CH}), 114.2(2 \times \mathrm{CH}), 65.0(\mathrm{CH}), 57.0(\mathrm{CH}), 55.4\left(\mathrm{CH}_{3}\right), 54.9\left(\mathrm{CH}_{2}\right)$, $52.5\left(\mathrm{CH}_{2}\right), 51.4\left(\mathrm{CH}_{3}\right), 46.0\left(\mathrm{CH}_{2}\right), 36.9\left(\mathrm{CH}_{2}\right)$. ESI-HRMS calculated for $\mathrm{C}_{15} \mathrm{H}_{20} \mathrm{NO}_{3} \mathrm{ClNa}[\mathrm{M}+\mathrm{Na}]^{+}$ 320.1029, found 320.1028. Trans 3j: Orange oil ${ }^{1} \mathrm{H}$ NMR $\left(\mathrm{CDCl}_{3}, 300 \mathrm{MHz}\right): \delta=7.27(\mathrm{~d}, J=8.7 \mathrm{~Hz}$, 
$2 \mathrm{H}), 6.86(\mathrm{~d}, J=8.7 \mathrm{~Hz}, 2 \mathrm{H}), 4.54$ (quint, $J=3.0 \mathrm{~Hz}, 1 \mathrm{H}), 3.87$ (dd, $J=11.0,2.4 \mathrm{~Hz}, 1 \mathrm{H}), 3.79$ (s, $3 \mathrm{H}), 3.63$ (s, $3 \mathrm{H}), 3.10(\mathrm{ABq}, 2 \mathrm{H}), 2.98-2.94(\mathrm{~m}, 2 \mathrm{H}), 2.32-2.21(\mathrm{~m}, 1 \mathrm{H}), 2.15-1.94(\mathrm{~m}, 3 \mathrm{H}) .{ }^{13} \mathrm{C}$ NMR $\left(\mathrm{CDCl}_{3}, 75 \mathrm{MHz}\right): \delta=171.6(\mathrm{C}), 159.2(\mathrm{C}), 134.4(\mathrm{C}), 129.0(2 \times \mathrm{CH}), 114.2(2 \times \mathrm{CH}), 59.9$ $(\mathrm{CH}), 57.6(\mathrm{CH}), 56.1\left(\mathrm{CH}_{2}\right), 55.4\left(\mathrm{CH}_{3}\right), 51.5\left(\mathrm{CH}_{3}\right), 47.6\left(\mathrm{CH}_{2}\right), 43.1\left(\mathrm{CH}_{2}\right), 33.6\left(\mathrm{CH}_{2}\right)$. ESI-HRMS calculated for $\mathrm{C}_{15} \mathrm{H}_{20} \mathrm{NO}_{3} \mathrm{ClNa}[\mathrm{M}+\mathrm{Na}]^{+} 320.1029$, found 320.1027 .

Methyl 2-(4-chloro-2-p-tolylpiperidin-1-yl)acetate $3 \boldsymbol{k}$ (74\%, $83 \mathrm{mg}$, dr: 62:38)

Cis 3k: Orange oil ${ }^{1} \mathrm{H}$ NMR $\left(\mathrm{CDCl}_{3}, 300 \mathrm{MHz}\right): \delta=7.23(\mathrm{~d}, J=7.9 \mathrm{~Hz}, 2 \mathrm{H}), 7.15(\mathrm{~d}, J=7.9 \mathrm{~Hz}, 2$ H), 3.94 (tt, $J=11.7,4.4 \mathrm{~Hz}, 1 \mathrm{H}), 3.61(\mathrm{~s}, 3 \mathrm{H}), 3.60(\mathrm{~d}, J=7.7 \mathrm{~Hz}, 1 \mathrm{H}), 3.12(\mathrm{dt}, J=11.8,3.4 \mathrm{~Hz}, 1$ H), $3.10(\mathrm{ABq}, 2 \mathrm{H}), 2.67(\mathrm{td}, J=12.0,2.1 \mathrm{~Hz}, 1 \mathrm{H}), 2.34(\mathrm{~s}, 3 \mathrm{H}), 2.25-2.18(\mathrm{~m}, 2 \mathrm{H}), 2.09$ (qd, $J=$ 11.9, $4.0 \mathrm{~Hz}, 1 \mathrm{H}), 2.00$ (q, $J=12.1 \mathrm{~Hz}, 1 \mathrm{H}) .{ }^{13} \mathrm{C} \mathrm{NMR}\left(\mathrm{CDCl}_{3}, 75 \mathrm{MHz}\right): \delta=171.3(\mathrm{C}), 138.8(\mathrm{C})$, $137.7(\mathrm{C}), 129.6(2 \times \mathrm{CH}), 127.6(2 \times \mathrm{CH}), 65.4(\mathrm{CH}), 56.9(\mathrm{CH}), 54.9\left(\mathrm{CH}_{2}\right), 52.5\left(\mathrm{CH}_{2}\right), 51.4\left(\mathrm{CH}_{3}\right)$, $45.9\left(\mathrm{CH}_{2}\right), 36.8\left(\mathrm{CH}_{2}\right), 21.2\left(\mathrm{CH}_{3}\right)$. ESI-HRMS calculated for $\mathrm{C}_{15} \mathrm{H}_{21} \mathrm{NO}_{2} \mathrm{Cl}[\mathrm{M}+\mathrm{H}]^{+} 282.1261$, found 282.1264. Trans 3k: Orange oil ${ }^{1} \mathrm{H}$ NMR $\left(\mathrm{CDCl}_{3}, 300 \mathrm{MHz}\right): \delta=7.24(\mathrm{~d}, J=7.9 \mathrm{~Hz}, 2 \mathrm{H}), 7.13(\mathrm{~d}, J$ $=7.9 \mathrm{~Hz}, 2 \mathrm{H}), 4.54$ (quint, $J=2.9 \mathrm{~Hz}, 1 \mathrm{H}), 3.87(\mathrm{dd}, J=10.8,2.9 \mathrm{~Hz}, 1 \mathrm{H}), 3.63(\mathrm{~s}, 3 \mathrm{H}), 3.11(\mathrm{ABq}$, $2 \mathrm{H}), 2.95-2.90(\mathrm{~m}, 2 \mathrm{H}), 2.32(\mathrm{~s}, 3 \mathrm{H}), 2.29-2.21(\mathrm{~m}, 1 \mathrm{H}), 2.15-2.06(\mathrm{~m}, 1 \mathrm{H}), 2.02-1.95(\mathrm{~m}, 2 \mathrm{H}) .{ }^{13} \mathrm{C}$ NMR $\left(\mathrm{CDCl}_{3}, 75 \mathrm{MHz}\right): \delta=171.6(\mathrm{C}), 139.5(\mathrm{C}), 137.4(\mathrm{C}), 129.5(2 \times \mathrm{CH}), 127.8(2 \times \mathrm{CH}), 60.3$ (CH), $57.5(\mathrm{CH}), 56.2\left(\mathrm{CH}_{2}\right), 51.5\left(\mathrm{CH}_{3}\right), 47.5\left(\mathrm{CH}_{2}\right), 43.1\left(\mathrm{CH}_{2}\right), 33.5\left(\mathrm{CH}_{2}\right), 21.2\left(\mathrm{CH}_{3}\right)$. ESI-HRMS calculated for $\mathrm{C}_{15} \mathrm{H}_{21} \mathrm{NO}_{2} \mathrm{Cl}[\mathrm{M}+\mathrm{H}]^{+} 282.1261$, found 282.1264.

Methyl 2-(4-chloro-2-isopropylpiperidin-1-yl)acetate 3 ( $86 \%, 80 \mathrm{mg}$, dr: 43:57)

Cis 31: Yellow oil ${ }^{1} \mathrm{H}$ NMR $\left(\mathrm{CDCl}_{3}, 300 \mathrm{MHz}\right): \delta=3.87(\mathrm{tt}, J=11.8,4.3 \mathrm{~Hz}, 1 \mathrm{H}), 3.69(\mathrm{~s}, 3 \mathrm{H}), 3.41$ (ABq, $2 \mathrm{H}), 2.91(\mathrm{ddd}, J=12.0,4.6,2.5 \mathrm{~Hz}, 1 \mathrm{H}), 2.77(\mathrm{td}, J=12.1,2.6 \mathrm{~Hz}, 1 \mathrm{H}), 2.55(\mathrm{ddd}, J=11.4$, 3.9, $2.0 \mathrm{~Hz}, 1 \mathrm{H}), 2.11-2.01(\mathrm{~m}, 2 \mathrm{H}), 1.96-1.79(\mathrm{~m}, 2 \mathrm{H}), 1.50(\mathrm{q}, J=11.9 \mathrm{~Hz}, 1 \mathrm{H}), 0.91(\mathrm{~d}, J=6.8$ $\mathrm{Hz}, 3 \mathrm{H}), 0.85(\mathrm{~d}, J=6.8 \mathrm{~Hz}, 3 \mathrm{H}) .{ }^{13} \mathrm{C} \mathrm{NMR}\left(\mathrm{CDCl}_{3}, 75 \mathrm{MHz}\right): \delta=171.6(\mathrm{C}), 63.4(\mathrm{CH}), 58.4(\mathrm{CH})$, 53.1 $\left(\mathrm{CH}_{2}\right), 52.9\left(\mathrm{CH}_{2}\right), 51.5\left(\mathrm{CH}_{3}\right), 36.6\left(\mathrm{CH}_{2}\right), 35.5\left(\mathrm{CH}_{2}\right), 27.9(\mathrm{CH}), 20.0\left(\mathrm{CH}_{3}\right), 15.2\left(\mathrm{CH}_{3}\right)$. ESIHRMS calculated for $\mathrm{C}_{11} \mathrm{H}_{21} \mathrm{NO}_{2} \mathrm{Cl}[\mathrm{M}+\mathrm{H}]^{+} 234.1261$, found 234.1263. Trans 31: Yellow oil ${ }^{1} \mathrm{H}$ NMR $\left(\mathrm{CDCl}_{3}, 300 \mathrm{MHz}\right): \delta=4.50$ (quint, $\left.J=3.6 \mathrm{~Hz}, 1 \mathrm{H}\right), 3.72(\mathrm{~s}, 3 \mathrm{H}), 3.42(\mathrm{ABq}, 2 \mathrm{H}), 3.09$ (td, $J=$ 11.8, $2.8 \mathrm{~Hz}, 1 \mathrm{H}), 2.85$ (ddd, $J=9.8,4.9,3.0 \mathrm{~Hz}, 1 \mathrm{H}), 2.76(\mathrm{dt}, J=12.0,4.1 \mathrm{~Hz}, 1 \mathrm{H}), 2.05-1.97$ (m, 
$2 \mathrm{H}), 1.87-1.81(\mathrm{~m}, 2 \mathrm{H}), 1.76(\mathrm{qd}, J=9.8,3.4 \mathrm{~Hz}, 1 \mathrm{H}), 0.90(\mathrm{~d}, J=6.8 \mathrm{~Hz}, 3 \mathrm{H}), 0.84(\mathrm{~d}, J=6.8 \mathrm{~Hz}$, $3 \mathrm{H}) .{ }^{13} \mathrm{C} \mathrm{NMR}\left(\mathrm{CDCl}_{3}, 75 \mathrm{MHz}\right): \delta=171.9(\mathrm{C}), 58.9(\mathrm{CH}), 57.9(\mathrm{CH}), 54.3\left(\mathrm{CH}_{2}\right), 51.6\left(\mathrm{CH}_{3}\right), 47.9$ $\left(\mathrm{CH}_{2}\right), 33.1\left(\mathrm{CH}_{2}\right), 32.1\left(\mathrm{CH}_{2}\right), 27.3(\mathrm{CH}), 19.9\left(\mathrm{CH}_{3}\right), 15.9\left(\mathrm{CH}_{3}\right)$. ESI-HRMS calculated for $\mathrm{C}_{11} \mathrm{H}_{21} \mathrm{NO}_{2} \mathrm{Cl}[\mathrm{M}+\mathrm{H}]^{+}$234.1261, found 234.1259.

Methyl 2-(4-chloro-2-phenethylpiperidin-1-yl)acetate $3 m$ (52\%, $61 \mathrm{mg}, \mathrm{dr}: 62: 38)$

Cis 3m: Orange oil ${ }^{1} \mathrm{H}$ NMR $\left(\mathrm{CDCl}_{3}, 300 \mathrm{MHz}\right): \delta=7.31-7.26$ (m, $\left.2 \mathrm{H}\right), 7.21-7.16$ (m, $\left.3 \mathrm{H}\right) 3.89$ (tt, $J$ = 11.7, 4.4 Hz, $1 \mathrm{H}), 3.68(\mathrm{~s}, 3 \mathrm{H}), 3.42(\mathrm{ABq}, 2 \mathrm{H}), 2.96(\mathrm{ddd}, J=12.2,4.2,2.9 \mathrm{~Hz}, 1 \mathrm{H}), 2.77-2.52$ (m, $4 \mathrm{H}$ ), 2.22 (dquint, $J=12.6,2.2 \mathrm{~Hz}, 1 \mathrm{H}), 2.09$ (dsex, $J=12.6,2.2 \mathrm{~Hz}, 1 \mathrm{H}), 1.93-1.86(\mathrm{~m}, 2 \mathrm{H})$, 1.77-1.65 (m, $2 \mathrm{H}) .{ }^{13} \mathrm{C} \mathrm{NMR}\left(\mathrm{CDCl}_{3}, 75 \mathrm{MHz}\right): \delta=171.4(\mathrm{C}), 142.0(\mathrm{C}), 128.6(2 \times \mathrm{CH}), 128.4(2 \times$ $\mathrm{CH}), 126.1(\mathrm{CH}), 58.5(\mathrm{CH}), 57.5(\mathrm{CH}), 53.2\left(\mathrm{CH}_{2}\right), 52.9\left(\mathrm{CH}_{2}\right), 51.6\left(\mathrm{CH}_{3}\right), 41.3\left(\mathrm{CH}_{2}\right), 36.2\left(\mathrm{CH}_{2}\right)$, $34.9\left(\mathrm{CH}_{2}\right), 31.0\left(\mathrm{CH}_{2}\right)$. ESI-HRMS calculated for $\mathrm{C}_{16} \mathrm{H}_{23} \mathrm{NO}_{2} \mathrm{Cl}[\mathrm{M}+\mathrm{H}]^{+} 296.1417$, found 296.1425. Trans 3m: Orange oil ${ }^{1} \mathrm{H}$ NMR $\left(\mathrm{CDCl}_{3}, 300 \mathrm{MHz}\right): \delta=7.31-7.26(\mathrm{~m}, 2 \mathrm{H}), 7.21-7.16(\mathrm{~m}, 3 \mathrm{H}), 4.43$ (quint, $J=4.2 \mathrm{~Hz}, 1 \mathrm{H}), 3.70(\mathrm{~s}, 3 \mathrm{H}), 3.40(\mathrm{ABq}, 2 \mathrm{H}), 3.06-2.95(\mathrm{~m}, 2 \mathrm{H}), 2.80-2.56(\mathrm{~m}, 3 \mathrm{H}), 2.09$ $2.02(\mathrm{~m}, 2 \mathrm{H}), 1.97-1.89(\mathrm{~m}, 3 \mathrm{H}), 1.77-1.70(\mathrm{~m}, 1 \mathrm{H}) .{ }^{13} \mathrm{C} \mathrm{NMR}\left(\mathrm{CDCl}_{3}, 75 \mathrm{MHz}\right): \delta=171.6(\mathrm{C})$, $142.1(\mathrm{C}), 128.6(2 \times \mathrm{CH}), 128.5(2 \times \mathrm{CH}), 126.0(\mathrm{CH}), 57.0(\mathrm{CH}), 54.8(\mathrm{CH}), 54.8\left(\mathrm{CH}_{2}\right), 51.8\left(\mathrm{CH}_{3}\right)$, $47.8\left(\mathrm{CH}_{2}\right), 38.1\left(\mathrm{CH}_{2}\right), 33.5\left(\mathrm{CH}_{2}\right), 33.0\left(\mathrm{CH}_{2}\right), 31.6\left(\mathrm{CH}_{2}\right)$. ESI-HRMS calculated for $\mathrm{C}_{16} \mathrm{H}_{23} \mathrm{NO}_{2} \mathrm{Cl}$ $[\mathrm{M}+\mathrm{H}]^{+}$296.1417, found 296.1414.

Methyl 2-(4-chloro-2-heptylpiperidin-1-yl)acetate 3n (78\%, 90 mg, dr: 62:38)

Cis 3n: Orange oil ${ }^{1} \mathrm{H}$ NMR ( $\left.\mathrm{CDCl}_{3}, 300 \mathrm{MHz}\right): \delta=3.88$ (tt, $\left.J=11.7,4.3 \mathrm{~Hz}, 1 \mathrm{H}\right), 3.70(\mathrm{~s}, 3 \mathrm{H}), 3.41$ (s, $2 \mathrm{H}), 2.94(\mathrm{dt}, J=12.1,3.0 \mathrm{~Hz}, 1 \mathrm{H}), 2.69(\mathrm{ddd}, J=12.1,4.2,2.8 \mathrm{~Hz}, 1 \mathrm{H}), 2.66-2.62(\mathrm{~m}, 1 \mathrm{H})$, 2.18-2.04 (m, $2 \mathrm{H}), 1.89$ (qd, $J=12.2,4.3 \mathrm{~Hz}, 1 \mathrm{H}), 1.60(\mathrm{q}, J=11.9 \mathrm{~Hz}, 1 \mathrm{H}), 1.26(\mathrm{~s}, 12 \mathrm{H}), 0.88$ (t, $J=6.6 \mathrm{~Hz}, 3 \mathrm{H}) .{ }^{13} \mathrm{C} \mathrm{NMR}\left(\mathrm{CDCl}_{3}, 75 \mathrm{MHz}\right): \delta=171.5(\mathrm{C}), 59.1(\mathrm{CH}), 57.7(\mathrm{CH}), 53.4\left(\mathrm{CH}_{2}\right), 53.0$ $\left(\mathrm{CH}_{2}\right), 51.6\left(\mathrm{CH}_{3}\right), 41.4\left(\mathrm{CH}_{2}\right), 36.3\left(\mathrm{CH}_{2}\right), 33.2\left(\mathrm{CH}_{2}\right), 31.9\left(\mathrm{CH}_{2}\right), 30.0\left(\mathrm{CH}_{2}\right), 29.3\left(\mathrm{CH}_{2}\right), 24.9$ $\left(\mathrm{CH}_{2}\right), 22.8\left(\mathrm{CH}_{2}\right), 14.2\left(\mathrm{CH}_{3}\right)$. ESI-HRMS calculated for $\mathrm{C}_{15} \mathrm{H}_{28} \mathrm{NO}_{2} \mathrm{ClNa}[\mathrm{M}+\mathrm{Na}]^{+}$312.1706, found 312.1714. Trans 3n: Orange oil ${ }^{1} \mathrm{H}$ NMR $\left(\mathrm{CDCl}_{3}, 300 \mathrm{MHz}\right): \delta=4.42$ (quint, $\left.J=4.0 \mathrm{~Hz}, 1 \mathrm{H}\right), 3.73$ (s, $3 \mathrm{H}), 3.42(\mathrm{ABq}, 2 \mathrm{H}), 3.05-2.98(\mathrm{~m}, 2 \mathrm{H}), 2.77$ (dt, $J=11.9,4.3 \mathrm{~Hz}, 1 \mathrm{H}), 2.11-2.07$ (m, $1 \mathrm{H}), 1.96-$ $1.85(\mathrm{~m}, 3 \mathrm{H}), 1.26(\mathrm{~s}, 12 \mathrm{H}), 0.88(\mathrm{t}, J=6.6 \mathrm{~Hz}, 3 \mathrm{H}) .{ }^{13} \mathrm{C} \mathrm{NMR}\left(\mathrm{CDCl}_{3}, 75 \mathrm{MHz}\right): \delta=171.5(\mathrm{C}), 57.1$ 
(CH), 55.1 (CH), $54.8\left(\mathrm{CH}_{2}\right), 51.8\left(\mathrm{CH}_{3}\right), 47.8\left(\mathrm{CH}_{2}\right), 38.2\left(\mathrm{CH}_{2}\right), 33.6\left(\mathrm{CH}_{2}\right), 31.9\left(\mathrm{CH}_{2}\right), 31.2\left(\mathrm{CH}_{2}\right)$, $30.0\left(\mathrm{CH}_{2}\right), 29.4\left(\mathrm{CH}_{2}\right), 25.5\left(\mathrm{CH}_{2}\right), 22.8\left(\mathrm{CH}_{2}\right), 14.2\left(\mathrm{CH}_{3}\right)$. ESI-HRMS calculated for $\mathrm{C}_{15} \mathrm{H}_{28} \mathrm{NO}_{2} \mathrm{ClNa}[\mathrm{M}+\mathrm{Na}]^{+}$312.1706, found 312.1706.

2-(4-bromophenyl)-4-chloro-1-(4-methoxyphenyl)piperidine 5a (99\%, $151 \mathrm{mg}, \mathrm{dr}: 12: 88)$

Cis 5a: Orange oil ${ }^{1} \mathrm{H}$ NMR $\left(\mathrm{CDCl}_{3}, 300 \mathrm{MHz}\right): \delta=7.27(\mathrm{~d}, J=8.9 \mathrm{~Hz}, 2 \mathrm{H}), 7.10(\mathrm{~d}, J=8.9 \mathrm{~Hz}, 2$ H), $6.86(\mathrm{~d}, J=8.3 \mathrm{~Hz}, 2 \mathrm{H}), 6.64(\mathrm{~d}, J=8.3 \mathrm{~Hz}, 2 \mathrm{H}), 4.02(\mathrm{tt}, J=11.7,4.3 \mathrm{~Hz}, 1 \mathrm{H}), 3.91(\mathrm{dd}, J=$ 11.1, $2.6 \mathrm{~Hz}, 1 \mathrm{H}), 3.67(\mathrm{~s}, 3 \mathrm{H}), 3.36(\mathrm{dt}, J=12.4,3.6 \mathrm{~Hz}, 1 \mathrm{H}), 2.79(\mathrm{td}, J=12.1,3.0 \mathrm{~Hz}, 1 \mathrm{H}), 2.37-$ $2.18(\mathrm{~m}, 2 \mathrm{H}), 2.17(\mathrm{qd}, J=12.1,4.1 \mathrm{~Hz}, 1 \mathrm{H}), 1.96(\mathrm{q}, J=12.1 \mathrm{~Hz}, 1 \mathrm{H}) .{ }^{13} \mathrm{C}$ NMR $\left(\mathrm{CDCl}_{3}, 75\right.$ MHz): $\delta=156.0(\mathrm{C}), 144.5(\mathrm{C}), 142.2(\mathrm{C}), 131.5(2 \times \mathrm{CH}), 129.3(2 \times \mathrm{CH}), 125.7(2 \times \mathrm{CH}), 120.5$ (C), $114.0(2 \times \mathrm{CH}), 64.6(\mathrm{CH}), 57.5\left(\mathrm{CH}_{2}\right), 56.8(\mathrm{CH}), 55.3\left(\mathrm{CH}_{3}\right), 46.9\left(\mathrm{CH}_{2}\right), 37.5\left(\mathrm{CH}_{2}\right)$. ESIHRMS calculated for $\mathrm{C}_{18} \mathrm{H}_{20} \mathrm{NOClBr}[\mathrm{M}+\mathrm{H}]^{+} 380.0417$, found 380.0418. Trans 5a: Black solid M.p. 106-108 ${ }^{\circ} \mathrm{C} .{ }^{1} \mathrm{H} \mathrm{NMR}\left(\mathrm{CDCl}_{3}, 300 \mathrm{MHz}\right): \delta=7.29(\mathrm{~d}, J=8.5 \mathrm{~Hz}, 2 \mathrm{H}), 7.13(\mathrm{~d}, J=8.5 \mathrm{~Hz}, 2 \mathrm{H}), 6.92$ $(\mathrm{d}, J=9.0 \mathrm{~Hz}, 2 \mathrm{H}), 6.68(\mathrm{~d}, J=9.0 \mathrm{~Hz}, 2 \mathrm{H}), 4.51-4.47(\mathrm{~m}, 2 \mathrm{H}), 3.70(\mathrm{~s}, 3 \mathrm{H}), 3.35(\mathrm{td}, J=10.9,2.8$ $\mathrm{Hz}, 1 \mathrm{H}), 3.16(\mathrm{dt}, J=12.3,4.1 \mathrm{~Hz}, 1 \mathrm{H}), 2.36-2.26(\mathrm{~m}, 1 \mathrm{H}), 2.17-2.14(\mathrm{~m}, 2 \mathrm{H}), 2.03(\mathrm{dd}, J=14.0$, $3.0 \mathrm{~Hz}, 1 \mathrm{H}) .{ }^{13} \mathrm{C} \mathrm{NMR}\left(\mathrm{CDCl}_{3}, 75 \mathrm{MHz}\right): \delta=155.4(\mathrm{C}), 145.2(\mathrm{C}), 142.3(\mathrm{C}), 131.5(2 \times \mathrm{CH}), 129.4$ $(2 \times \mathrm{CH}), 124.4(2 \times \mathrm{CH}), 120.4(\mathrm{C}), 114.1(2 \times \mathrm{CH}), 58.7(\mathrm{CH}), 56.9(\mathrm{CH}), 55.4\left(\mathrm{CH}_{3}\right), 50.7\left(\mathrm{CH}_{2}\right)$, $43.4\left(\mathrm{CH}_{2}\right), 34.5\left(\mathrm{CH}_{2}\right)$. ESI-HRMS calculated for $\mathrm{C}_{18} \mathrm{H}_{20} \mathrm{NOClBr}[\mathrm{M}+\mathrm{H}]^{+} 380.0417$, found 380.0416 . 4-chloro-1-(4-methoxyphenyl)-2-phenylpiperidine $5 \boldsymbol{b}$ (93\%, $113 \mathrm{mg}$, dr: 23:77)

Cis 5b: Yellow solid M.p. $78-80{ }^{\circ} \mathrm{C} .{ }^{1} \mathrm{H}$ NMR $\left(\mathrm{CDCl}_{3}, 300 \mathrm{MHz}\right): \delta=7.26-7.06(\mathrm{~m}, 5 \mathrm{H}), 6.89(\mathrm{~d}, J=$ $9.0 \mathrm{~Hz}, 2 \mathrm{H}), 6.63(\mathrm{~d}, J=9.0 \mathrm{~Hz}, 2 \mathrm{H}), 4.01(\mathrm{tt}, J=11.4,4.5 \mathrm{~Hz}, 1 \mathrm{H}), 3.93(\mathrm{dd}, J=11.1,2.7 \mathrm{~Hz}, 1 \mathrm{H})$, $3.65(\mathrm{~s}, 3 \mathrm{H}), 3.39(\mathrm{dt}, J=12.4,3.5 \mathrm{~Hz}, 1 \mathrm{H}), 2.81(\mathrm{td}, J=12.1,3.1 \mathrm{~Hz}, 1 \mathrm{H}), 2.40-2.34(\mathrm{~m}, 1 \mathrm{H})$, 2.25-2.16 (m, $2 \mathrm{H}), 2.02(\mathrm{q}, J=11.9 \mathrm{~Hz}, 1 \mathrm{H}) .{ }^{13} \mathrm{C} \mathrm{NMR}\left(\mathrm{CDCl}_{3}, 75 \mathrm{MHz}\right): \delta=155.8(\mathrm{C}), 144.9(\mathrm{C})$, $143.1(\mathrm{C}), 128.3(2 \times \mathrm{CH}), 127.6(2 \times \mathrm{CH}), 126.9(\mathrm{CH}), 125.7(2 \times \mathrm{CH}), 113.9(2 \times \mathrm{CH}), 65.3(\mathrm{CH})$, 57.6 $\left(\mathrm{CH}_{2}\right), 57.1(\mathrm{CH}), 55.3\left(\mathrm{CH}_{3}\right), 47.1\left(\mathrm{CH}_{2}\right), 37.6\left(\mathrm{CH}_{2}\right)$. ESI-HRMS calculated for $\mathrm{C}_{18} \mathrm{H}_{21} \mathrm{NOCl}$ $[\mathrm{M}+\mathrm{H}]^{+}$302.1306, found 302.1305. Trans 5b: Orange oil ${ }^{1} \mathrm{H}$ NMR $\left(\mathrm{CDCl}_{3}, 300 \mathrm{MHz}\right): \delta=7.27-7.08$ $(\mathrm{m}, 5 \mathrm{H}), 6.92(\mathrm{~d}, J=9.0 \mathrm{~Hz}, 2 \mathrm{H}), 6.67(\mathrm{~d}, J=9.0 \mathrm{~Hz}, 2 \mathrm{H}), 4.57(\mathrm{dd}, J=8.5,3.9 \mathrm{~Hz}, 1 \mathrm{H}), 4.46$ (quint, $J=4.1 \mathrm{~Hz}, 1 \mathrm{H}), 3.66(\mathrm{~s}, 3 \mathrm{H}), 3.39(\mathrm{td}, J=11.3,2.9 \mathrm{~Hz}, 1 \mathrm{H}), 3.18(\mathrm{dt}, J=12.6,4.4 \mathrm{~Hz}, 1 \mathrm{H})$, 
2.35-2.14 (m, $3 \mathrm{H}), 2.06-1.99$ (m, $1 \mathrm{H}) .{ }^{13} \mathrm{C}$ NMR ( $\left.\mathrm{CDCl}_{3}, 75 \mathrm{MHz}\right): \delta=154.9(\mathrm{C}), 145.4(\mathrm{C}), 142.8$ (C), $128.4(2 \times \mathrm{CH}), 127.6(2 \times \mathrm{CH}), 126.7(\mathrm{C}), 123.6(2 \times \mathrm{CH}), 114.1(2 \times \mathrm{CH}), 59.2(\mathrm{CH}), 57.0$ (CH), $55.4\left(\mathrm{CH}_{3}\right), 50.1\left(\mathrm{CH}_{2}\right), 43.1\left(\mathrm{CH}_{2}\right), 34.6\left(\mathrm{CH}_{2}\right)$. ESI-HRMS calculated for $\mathrm{C}_{18} \mathrm{H}_{21} \mathrm{NOCl}[\mathrm{M}+\mathrm{H}]^{+}$ 302.1306, found 302.1306 .

4-chloro-2-(2-chlorophenyl)-1-(4-methoxyphenyl)piperidine 5c (99\%, $130 \mathrm{mg}$, dr: 28:72)

Cis 5c: Yellow oil ${ }^{1} \mathrm{H}$ NMR ( $\left.\mathrm{CDCl}_{3}, 300 \mathrm{MHz}\right): \delta=7.45-7.41$ (m, $\left.1 \mathrm{H}\right)$, 7.23-7.20 (m, $\left.1 \mathrm{H}\right)$, 7.04-6.95 (m, $2 \mathrm{H}), 6.87$ (d, $J=9.0 \mathrm{~Hz}, 2 \mathrm{H}), 6.65(\mathrm{~d}, J=9.0 \mathrm{~Hz}, 2 \mathrm{H}), 4.50(\mathrm{dd}, J=11.0,2.7 \mathrm{~Hz}, 1 \mathrm{H}), 4.06$ (tt, $J=11.6,4.5 \mathrm{~Hz}, 1 \mathrm{H}), 3.67(\mathrm{~s}, 3 \mathrm{H}), 3.45(\mathrm{dt}, J=12.4,3.6 \mathrm{~Hz}, 1 \mathrm{H}), 2.79(\mathrm{td}, J=12.2,3.0 \mathrm{~Hz}, 1 \mathrm{H})$, 2.48-2.43 (m, $1 \mathrm{H}), 2.27-2.24(\mathrm{~m}, 1 \mathrm{H}), 2.19(\mathrm{qd}, J=12.0,4.2 \mathrm{~Hz}, 1 \mathrm{H}), 1.85(\mathrm{q}, J=12.0 \mathrm{~Hz}, 1 \mathrm{H}) .{ }^{13} \mathrm{C}$ NMR (CDCl 3 , $75 \mathrm{MHz}): \delta=155.8(\mathrm{C}), 144.8(\mathrm{C}), 140.1(\mathrm{C}), 132.2(\mathrm{C}), 129.3(\mathrm{CH}), 129.2(\mathrm{CH})$, $127.9(\mathrm{CH}), 127.2(\mathrm{CH}), 124.9(2 \times \mathrm{CH}), 114.1(2 \times \mathrm{CH}), 59.7(\mathrm{CH}), 58.0\left(\mathrm{CH}_{2}\right), 56.8(\mathrm{CH}), 55.3$ $\left(\mathrm{CH}_{3}\right), 44.8\left(\mathrm{CH}_{2}\right), 37.6\left(\mathrm{CH}_{2}\right)$. ESI-HRMS calculated for $\mathrm{C}_{18} \mathrm{H}_{20} \mathrm{NOCl}_{2}[\mathrm{M}+\mathrm{H}]^{+} 336.0922$, found 336.0923. Trans 5c: Yellow solid M.p. $96-98{ }^{\circ} \mathrm{C} .{ }^{1} \mathrm{H}$ NMR $\left(\mathrm{CDCl}_{3}, 300 \mathrm{MHz}\right): \delta=7.42-7.38(\mathrm{~m}, 1$ H), 7.23-7.20 (m, $1 \mathrm{H}), 7.01-6.97(\mathrm{~m}, 2 \mathrm{H}), 6.92(\mathrm{~d}, J=9.0 \mathrm{~Hz}, 2 \mathrm{H}), 6.66(\mathrm{~d}, J=9.0 \mathrm{~Hz}, 2 \mathrm{H}), 5.00$ (dd, $J=10.4,2.9 \mathrm{~Hz}, 1 \mathrm{H}), 4.55$ (quint, $J=3.2 \mathrm{~Hz}, 1 \mathrm{H}$ ), $3.65(\mathrm{~s}, 3 \mathrm{H}), 3.32-3.26(\mathrm{~m}, 2 \mathrm{H}), 2.37-2.23$ (m, $2 \mathrm{H}), 2.08-1.92$ (m, $2 \mathrm{H}) .{ }^{13} \mathrm{C} \mathrm{NMR}\left(\mathrm{CDCl}_{3}, 75 \mathrm{MHz}\right): \delta=155.5(\mathrm{C}), 145.5(\mathrm{C}), 140.7(\mathrm{C}), 132.5$ (C), $129.3(\mathrm{CH}), 129.3(\mathrm{CH}), 127.7(\mathrm{CH}), 127.0(\mathrm{CH}), 124.5(2 \times \mathrm{CH}), 114.1(2 \times \mathrm{CH}), 56.9(\mathrm{CH})$, $55.3\left(\mathrm{CH}_{3}\right), 54.3(\mathrm{CH}), 52.1\left(\mathrm{CH}_{2}\right), 41.9\left(\mathrm{CH}_{2}\right), 34.4\left(\mathrm{CH}_{2}\right)$. ESI-HRMS calculated for $\mathrm{C}_{18} \mathrm{H}_{20} \mathrm{NOCl}_{2}$ $[\mathrm{M}+\mathrm{H}]^{+} 336.0922$, found 336.0919 .

4-chloro-1-(4-methoxyphenyl)-2-(4-nitrophenyl)piperidine 5d (92\%, $131 \mathrm{mg}$, dr: 14:86)

Trans 5d: Black solid M.p. 106-108 ${ }^{\circ} \mathrm{C} .{ }^{1} \mathrm{H}$ NMR $\left(\mathrm{CDCl}_{3}, 300 \mathrm{MHz}\right): \delta=8.02(\mathrm{~d}, J=8.7 \mathrm{~Hz}, 2 \mathrm{H})$, $7.45(\mathrm{~d}, J=8.7 \mathrm{~Hz}, 2 \mathrm{H}), 6.94(\mathrm{~d}, J=8.9 \mathrm{~Hz}, 2 \mathrm{H}), 6.68(\mathrm{~d}, J=8.9 \mathrm{~Hz}, 2 \mathrm{H}), 4.62(\mathrm{dd}, J=9.6,3.7 \mathrm{~Hz}$, $1 \mathrm{H}), 4.56$ (quint, $J=3.3 \mathrm{~Hz}, 1 \mathrm{H}), 3.68(\mathrm{~s}, 3 \mathrm{H}), 3.34(\mathrm{td}, J=11.9,2.7 \mathrm{~Hz}, 1 \mathrm{H}), 3.19$ (dt, $J=12.3,3.9$ $\mathrm{Hz}, 1 \mathrm{H}), 2.39-2.30$ (m, $1 \mathrm{H}), 2.18-2.05$ (m, $3 \mathrm{H}) .{ }^{13} \mathrm{C} \mathrm{NMR}\left(\mathrm{CDCl}_{3}, 75 \mathrm{MHz}\right): \delta=155.9(\mathrm{C}), 151.3$ (C), $146.8(\mathrm{C}), 144.9(\mathrm{C}), 128.5(2 \times \mathrm{CH}), 125.0(2 \times \mathrm{CH}), 123.7(2 \times \mathrm{CH}), 114.2(2 \times \mathrm{CH}), 58.8$ $(\mathrm{CH}), 56.6(\mathrm{CH}), 55.4\left(\mathrm{CH}_{3}\right), 51.2\left(\mathrm{CH}_{2}\right), 43.5\left(\mathrm{CH}_{2}\right), 34.2\left(\mathrm{CH}_{2}\right)$. ESI-HRMS calculated for $\mathrm{C}_{18} \mathrm{H}_{20} \mathrm{~N}_{2} \mathrm{O}_{3} \mathrm{Cl}[\mathrm{M}+\mathrm{H}]^{+}$347.1157, found 347.1160. 
4-chloro-1,2-bis(4-methoxyphenyl)piperidine 5e (40\%, $52 \mathrm{mg}$, dr: 20:80)

Trans 5e: Orange solid M.p. $98-100{ }^{\circ} \mathrm{C} .{ }^{1} \mathrm{H}$ NMR $\left(\mathrm{CDCl}_{3}, 300 \mathrm{MHz}\right): \delta=7.16(\mathrm{~d}, J=8.8 \mathrm{~Hz}, 2 \mathrm{H})$, $6.92(\mathrm{~d}, J=8.8 \mathrm{~Hz}, 2 \mathrm{H}), 6.72(\mathrm{~d}, J=8.8 \mathrm{~Hz}, 2 \mathrm{H}), 6.68(\mathrm{~d}, J=8.8 \mathrm{~Hz}, 2 \mathrm{H}), 4.53-4.46(\mathrm{~m}, 2 \mathrm{H}), 3.71$ (s, $3 \mathrm{H}), 3.68$ (s, $3 \mathrm{H}), 3.38$ (td, $J=10.5,2.7 \mathrm{~Hz}, 1 \mathrm{H}), 3.17$ (dt, $J=12.5,4.4 \mathrm{~Hz}, 1 \mathrm{H}), 2.29-2.17$ (m, 3 $\mathrm{H}), 2.05-1.99(\mathrm{~m}, 1 \mathrm{H}) .{ }^{13} \mathrm{C} \mathrm{NMR}\left(\mathrm{CDCl}_{3}, 75 \mathrm{MHz}\right): \delta=155.3(\mathrm{C}), 155.0(\mathrm{C}), 145.5(\mathrm{C}), 134.8(\mathrm{C})$, $128.7(2 \times \mathrm{CH}), 123.7(2 \times \mathrm{CH}), 114.0(2 \times \mathrm{CH}), 113.7(2 \times \mathrm{CH}), 58.6(\mathrm{CH}), 57.1(\mathrm{CH}), 55.4\left(\mathrm{CH}_{3}\right)$, $55.2\left(\mathrm{CH}_{3}\right), 50.0\left(\mathrm{CH}_{2}\right), 43.1\left(\mathrm{CH}_{2}\right), 34.7\left(\mathrm{CH}_{2}\right)$. ESI-HRMS calculated for $\mathrm{C}_{19} \mathrm{H}_{23} \mathrm{NO}_{2} \mathrm{Cl}[\mathrm{M}+\mathrm{H}]^{+}$ 332.1417, found 332.1417.

4-chloro-1-(4-methoxyphenyl)-2-p-tolylpiperidine $5 f(90 \%, 113 \mathrm{mg}$, dr: 22:78)

Cis 5f: Orange oil ${ }^{1} \mathrm{H}$ NMR $\left(\mathrm{CDCl}_{3}, 300 \mathrm{MHz}\right): \delta=7.10(\mathrm{~d}, J=7.9 \mathrm{~Hz}, 2 \mathrm{H}), 6.95(\mathrm{~d}, J=7.9 \mathrm{~Hz}, 2$ H), $6.89(\mathrm{~d}, J=9.0 \mathrm{~Hz}, 2 \mathrm{H}), 6.63(\mathrm{~d}, J=9.0 \mathrm{~Hz}, 2 \mathrm{H}), 4.03(\mathrm{tt}, J=11.4,4.7 \mathrm{~Hz}, 1 \mathrm{H}), 3.89(\mathrm{dd}, J=$ 11.1, $2.7 \mathrm{~Hz}, 1 \mathrm{H}), 3.66(\mathrm{~s}, 3 \mathrm{H}), 3.38(\mathrm{dt}, J=12.3,3.5 \mathrm{~Hz}, 1 \mathrm{H}), 2.79(\mathrm{td}, J=12.0,3.1 \mathrm{~Hz}, 1 \mathrm{H}), 2.38-$ $2.32(\mathrm{~m}, 1 \mathrm{H}), 2.24-2.15(\mathrm{~m}, 5 \mathrm{H}), 2.01(\mathrm{q}, J=12.0 \mathrm{~Hz}, 1 \mathrm{H}) .{ }^{13} \mathrm{C} \mathrm{NMR}\left(\mathrm{CDCl}_{3}, 75 \mathrm{MHz}\right): \delta=155.8$ (C), $145.0(\mathrm{C}), 140.1(\mathrm{C}), 136.4(\mathrm{C}), 129.1(2 \times \mathrm{CH}), 127.4(2 \times \mathrm{CH}), 125.7(2 \times \mathrm{CH}), 113.8(2 \times \mathrm{CH})$, $64.9(\mathrm{CH}), 57.6\left(\mathrm{CH}_{2}\right), 57.2(\mathrm{CH}), 55.3\left(\mathrm{CH}_{3}\right), 47.2\left(\mathrm{CH}_{2}\right), 37.6\left(\mathrm{CH}_{2}\right), 21.2\left(\mathrm{CH}_{3}\right)$. ESI-HRMS calculated for $\mathrm{C}_{19} \mathrm{H}_{23} \mathrm{NOCl}[\mathrm{M}+\mathrm{H}]^{+}$316.1468, found 316.1465. Trans 5f: Orange solid M.p. 102-104 ${ }^{\circ} \mathrm{C} .{ }^{1} \mathrm{H}$ NMR $\left(\mathrm{CDCl}_{3}, 300 \mathrm{MHz}\right): \delta=7.14(\mathrm{~d}, J=7.9 \mathrm{~Hz}, 2 \mathrm{H}), 6.98(\mathrm{~d}, J=7.9 \mathrm{~Hz}, 2 \mathrm{H}), 6.92(\mathrm{~d}, J=$ $9.0 \mathrm{~Hz}, 2 \mathrm{H}), 6.68(\mathrm{~d}, J=9.0 \mathrm{~Hz}, 2 \mathrm{H}), 4.55(\mathrm{dd}, J=8.5,3.7 \mathrm{~Hz}, 1 \mathrm{H}), 4.44$ (quint, $J=4.7 \mathrm{~Hz}, 1 \mathrm{H}$ ), $3.66(\mathrm{~s}, 3 \mathrm{H}), 3.38(\mathrm{td}, J=10.0,3.0 \mathrm{~Hz}, 1 \mathrm{H}), 3.18(\mathrm{dt}, J=12.6,4.6 \mathrm{~Hz}, 1 \mathrm{H}), 2.29-2.13(\mathrm{~m}, 6 \mathrm{H})$, 2.04-2.00 (m, $1 \mathrm{H}) .{ }^{13} \mathrm{C}$ NMR $\left(\mathrm{CDCl}_{3}, 75 \mathrm{MHz}\right): \delta=155.8(\mathrm{C}), 145.5(\mathrm{C}), 139.7(\mathrm{C}), 136.2(\mathrm{C}), 129.1$ $(2 \times \mathrm{CH}), 127.5(2 \times \mathrm{CH}), 123.4(2 \times \mathrm{CH}), 114.1(2 \times \mathrm{CH}), 58.9(\mathrm{CH}), 57.0(\mathrm{CH}), 55.4\left(\mathrm{CH}_{3}\right), 49.9$ $\left(\mathrm{CH}_{2}\right), 43.1\left(\mathrm{CH}_{2}\right), 34.7\left(\mathrm{CH}_{2}\right), 21.1\left(\mathrm{CH}_{3}\right)$. ESI-HRMS calculated for $\mathrm{C}_{19} \mathrm{H}_{23} \mathrm{NOCl}[\mathrm{M}+\mathrm{H}]^{+} 316.1468$, found 316.1468 .

4-chloro-2-isopropyl-1-(4-methoxyphenyl)piperidine 5g (98\%, $105 \mathrm{mg}$, dr: 9:91)

Cis 5g: Orange oil ${ }^{1} \mathrm{H}$ NMR $\left(\mathrm{CDCl}_{3}, 300 \mathrm{MHz}\right): \delta=7.07(\mathrm{~d}, J=8.9 \mathrm{~Hz}, 2 \mathrm{H}), 6.84(\mathrm{~d}, J=8.9 \mathrm{~Hz}, 2$ H), $3.99(\mathrm{tt}, J=11.5,4.3 \mathrm{~Hz}, 1 \mathrm{H}), 3.79(\mathrm{~s}, 3 \mathrm{H}), 3.07(\mathrm{dt}, J=12.2,3.6 \mathrm{~Hz}, 1 \mathrm{H}), 2.75-2.67(\mathrm{~m}, 2 \mathrm{H})$, 2.15-2.13 (m, $2 \mathrm{H}), 1.99$ (qd, $J=12.0,4.3 \mathrm{~Hz}, 1 \mathrm{H}), 1.71(\mathrm{q}, J=11.5 \mathrm{~Hz}, 1 \mathrm{H}), 1-70-1.65(\mathrm{~m}, 1 \mathrm{H})$, 
$0.78(\mathrm{t}, J=7.2 \mathrm{~Hz}, 6 \mathrm{H}) .{ }^{13} \mathrm{C} \mathrm{NMR}\left(\mathrm{CDCl}_{3}, 75 \mathrm{MHz}\right): \delta=156.9(\mathrm{C}), 144.8(\mathrm{C}), 126.5(2 \times \mathrm{CH}), 114.4$ $(2 \times \mathrm{CH}), 65.0(\mathrm{CH}), 58.7(\mathrm{CH}), 56.9\left(\mathrm{CH}_{2}\right), 55.5\left(\mathrm{CH}_{3}\right), 37.5\left(\mathrm{CH}_{2}\right), 35.7\left(\mathrm{CH}_{2}\right), 28.2(\mathrm{CH}), 19.7$ $\left(\mathrm{CH}_{3}\right), 15.1\left(\mathrm{CH}_{3}\right)$. ESI-HRMS calculated for $\mathrm{C}_{15} \mathrm{H}_{23} \mathrm{NOCl}[\mathrm{M}+\mathrm{H}]^{+}$268.1468, found 268.1465. Trans 5g: Orange oil ${ }^{1} \mathrm{H} \mathrm{NMR}\left(\mathrm{CDCl}_{3}, 300 \mathrm{MHz}\right): \delta=6.93(\mathrm{~d}, J=9.1 \mathrm{~Hz}, 2 \mathrm{H}), 6.82(\mathrm{~d}, J=9.1 \mathrm{~Hz}, 2 \mathrm{H})$, $4.37(\mathrm{sp}, J=4.1 \mathrm{~Hz} 1 \mathrm{H}), 3.76(\mathrm{~s}, 3 \mathrm{H}), 3.44(\mathrm{dt}, J=13.8,4.7 \mathrm{~Hz}, 1 \mathrm{H}), 3.30$ (m, $1 \mathrm{H}), 3.03(\mathrm{~m}, 1 \mathrm{H})$, 2.13-2.07 (m, $2 \mathrm{H}), 1.96-1.86(\mathrm{~m}, 3 \mathrm{H}), 0.89$ (d, $J=6.7 \mathrm{~Hz}, 3 \mathrm{H}), 0.85(\mathrm{~d}, J=6.7 \mathrm{~Hz}, 3 \mathrm{H}) .{ }^{13} \mathrm{C} \mathrm{NMR}$ $\left(\mathrm{CDCl}_{3}, 75 \mathrm{MHz}\right): \delta=153.8(\mathrm{C}), 145.3(\mathrm{C}), 120.4(2 \times \mathrm{CH}), 114.6(2 \times \mathrm{CH}), 62.5(\mathrm{CH}), 56.7(\mathrm{CH})$, $55.7\left(\mathrm{CH}_{3}\right), 46.0\left(\mathrm{CH}_{2}\right), 34.7\left(\mathrm{CH}_{2}\right), 34.5\left(\mathrm{CH}_{2}\right), 27.5(\mathrm{CH}), 20.4\left(\mathrm{CH}_{3}\right), 18.6\left(\mathrm{CH}_{3}\right)$. ESI-HRMS calculated for $\mathrm{C}_{15} \mathrm{H}_{23} \mathrm{NOCl}[\mathrm{M}+\mathrm{H}]^{+} 268.1468$, found 268.1466 .

2-(4-bromophenyl)-4-chloropiperidine cis 7a Yellow solid (77\%, $84 \mathrm{mg}$ )

M.p. $64-66{ }^{\circ} \mathrm{C} .{ }^{1} \mathrm{H} \mathrm{NMR}\left(\mathrm{CDCl}_{3}, 300 \mathrm{MHz}\right): \delta=7.45(\mathrm{~d}, J=8.4 \mathrm{~Hz}, 2 \mathrm{H}), 7.23(\mathrm{~d}, J=8.4 \mathrm{~Hz}, 2 \mathrm{H})$, 3.99 (tt, $J=11.7,4.3 \mathrm{~Hz}, 1 \mathrm{H}), 3.61(\mathrm{dd}, J=11.4,2.5 \mathrm{~Hz}, 1 \mathrm{H}), 3.23(\mathrm{ddd}, J=12.2,2.5,1.7 \mathrm{~Hz}, 1 \mathrm{H})$, $2.79(\mathrm{td}, J=12.2,2.5 \mathrm{~Hz}, 1 \mathrm{H}), 2.30-2.17(\mathrm{~m}, 2 \mathrm{H}), 1.84(\mathrm{ddd}, J=12.2,4.6,2.3 \mathrm{~Hz}, 1 \mathrm{H}), 1.82(\mathrm{~s}$, $\mathrm{NH}), 1.76(\mathrm{q}, J=12.0 \mathrm{~Hz}, 1 \mathrm{H}) .{ }^{13} \mathrm{C} \mathrm{NMR}\left(\mathrm{CDCl}_{3}, 75 \mathrm{MHz}\right): \delta=142.4(\mathrm{C}), 131.8(2 \times \mathrm{CH}), 128.5(2$ $\times \mathrm{CH}), 121.4(\mathrm{C}), 61.0(\mathrm{CH}), 57.4(\mathrm{CH}), 46.4\left(\mathrm{CH}_{2}\right), 45.3\left(\mathrm{CH}_{2}\right), 36.8\left(\mathrm{CH}_{2}\right)$. ESI-HRMS calculated for $\mathrm{C}_{11} \mathrm{H}_{14} \mathrm{NClBr}[\mathrm{M}+\mathrm{H}]^{+}$273.9998, found 273.9998.

4-chloro-2-phenylpiperidine cis $\mathbf{7 b}^{10 \mathrm{a}}$ Yellow solid (86\%, $68 \mathrm{mg}$ )

M.p. $60-62{ }^{\circ} \mathrm{C} .{ }^{1} \mathrm{H} \mathrm{NMR}\left(\mathrm{CDCl}_{3}, 300 \mathrm{MHz}\right): \delta=7.37-7.29(\mathrm{~m}, 5 \mathrm{H}), 4.04(\mathrm{tt}, J=11.8,4.4 \mathrm{~Hz}, 1 \mathrm{H})$, $3.66(\mathrm{dd}, J=11.4,2.4 \mathrm{~Hz}, 1 \mathrm{H}), 3.26(\mathrm{ddd}, J=12.2,4.4,2.4 \mathrm{~Hz}, 1 \mathrm{H}), 2.66(\mathrm{td}, J=12.2,2.4 \mathrm{~Hz}, 1 \mathrm{H})$, 2.34 (dquint, $J=12.6,2.3 \mathrm{~Hz}, 1 \mathrm{H}), 2.22$ (dsex, $J=12.6,2.3 \mathrm{~Hz}, 1 \mathrm{H}), 1.90-1.88$ (m, $3 \mathrm{H}) .{ }^{13} \mathrm{C}$ NMR $\left(\mathrm{CDCl}_{3}, 75 \mathrm{MHz}\right): \delta=143.4(\mathrm{C}), 128.7(2 \times \mathrm{CH}), 127.7(\mathrm{CH}), 126.7(2 \times \mathrm{CH}), 61.6(\mathrm{CH}), 57.7(\mathrm{CH})$, $46.5\left(\mathrm{CH}_{2}\right), 45.4\left(\mathrm{CH}_{2}\right), 37.0\left(\mathrm{CH}_{2}\right)$. ESI-HRMS calculated for $\mathrm{C}_{11} \mathrm{H}_{15} \mathrm{NCl}[\mathrm{M}+\mathrm{H}]^{+} 196.0893$, found 196.0897.

4-chloro-2-(2-chlorophenyl)piperidine cis 7c Orange oil (80\%, $74 \mathrm{mg})$

${ }^{1} \mathrm{H} \mathrm{NMR}\left(\mathrm{CDCl}_{3}, 300 \mathrm{MHz}\right): \delta=7.62(\mathrm{dd}, J=7.7,1.7 \mathrm{~Hz}, 1 \mathrm{H}), 7.36-7.17(\mathrm{~m}, 3 \mathrm{H}), 4.14(\mathrm{dd}, J=$ 11.3, $2.0 \mathrm{~Hz}, 1 \mathrm{H}), 4.03(\mathrm{tt}, J=11.7,4.3 \mathrm{~Hz}, 1 \mathrm{H}), 3.41(\mathrm{bs}, \mathrm{NH}), 3.26(\mathrm{ddd}, J=12.2,4.1,2.5 \mathrm{~Hz}, 1$ H), $2.85(\mathrm{td}, J=12.2,2.5 \mathrm{~Hz}, 1 \mathrm{H}), 2.40$ (dquint, $J=12.6,2.1 \mathrm{~Hz}, 1 \mathrm{H}), 2.22(\mathrm{dsex}, J=12.6,2.1 \mathrm{~Hz}, 1$ 
$\mathrm{H}), 1.93(\mathrm{qd}, J=12.1,4.3 \mathrm{~Hz}, 1 \mathrm{H}), 1.76(\mathrm{q}, J=12.1 \mathrm{~Hz}, 1 \mathrm{H}) .{ }^{13} \mathrm{C} \mathrm{NMR}\left(\mathrm{CDCl}_{3}, 75 \mathrm{MHz}\right): \delta=139.7$ (C), $132.7(\mathrm{C}), 129.7(\mathrm{CH}), 128.8(\mathrm{CH}), 127.7(\mathrm{CH}), 127.5(\mathrm{CH}), 57.3(\mathrm{CH}), 56.9(\mathrm{CH}), 46.3\left(\mathrm{CH}_{2}\right)$, $43.1\left(\mathrm{CH}_{2}\right), 36.4\left(\mathrm{CH}_{2}\right)$. ESI-HRMS calculated for $\mathrm{C}_{11} \mathrm{H}_{14} \mathrm{NCl}_{2}[\mathrm{M}+\mathrm{H}]^{+} 230.0503$, found 230.0503 . 4-chloro-2-(4-nitrophenyl)piperidine cis $\mathbf{7 d}^{10 \mathrm{a}}$ Orange solid (82\%, $\left.79 \mathrm{mg}\right)$

M.p. $100-102{ }^{\circ} \mathrm{C} .{ }^{1} \mathrm{H} \mathrm{NMR}\left(\mathrm{CDCl}_{3}, 300 \mathrm{MHz}\right): \delta=8.20(\mathrm{~d}, J=8.6 \mathrm{~Hz}, 2 \mathrm{H}), 7.56(\mathrm{~d}, J=8.6 \mathrm{~Hz}, 2 \mathrm{H})$, 4.02 (tt, $J=11.8,4.4 \mathrm{~Hz}, 1 \mathrm{H}), 3.79(\mathrm{dd}, J=11.3,2.4 \mathrm{~Hz}, 1 \mathrm{H}), 3.28(\mathrm{ddd}, J=12.2,4.3,2.6 \mathrm{~Hz}, 1 \mathrm{H})$, $2.84(\mathrm{td}, J=12.2,2.6 \mathrm{~Hz}, 1 \mathrm{H}), 2.35-2.20(\mathrm{~m}, 2 \mathrm{H}), 1.94(\mathrm{bs}, \mathrm{NH}), 1.87(\mathrm{qd}, J=12.2,4.5 \mathrm{~Hz}, 1 \mathrm{H})$, $1.77(\mathrm{q}, J=12.2 \mathrm{~Hz}, 1 \mathrm{H}) .{ }^{13} \mathrm{C} \mathrm{NMR}\left(\mathrm{CDCl}_{3}, 75 \mathrm{MHz}\right): \delta=150.8(\mathrm{C}), 147.5(\mathrm{C}), 127.6(2 \times \mathrm{CH})$, 124.0 $(2 \times \mathrm{CH}), 61.0(\mathrm{CH}), 57.0(\mathrm{CH}), 46.3\left(\mathrm{CH}_{2}\right), 45.3\left(\mathrm{CH}_{2}\right), 36.7\left(\mathrm{CH}_{2}\right)$. ESI-HRMS calculated for $\mathrm{C}_{11} \mathrm{H}_{14} \mathrm{~N}_{2} \mathrm{O}_{2} \mathrm{Cl}[\mathrm{M}+\mathrm{H}]^{+} 241.0744$, found 241.0747.

4-chloro-2-(4-methoxyphenyl)piperidine cis $7 \mathbf{e}^{10 \mathrm{a}}$ White solid (34\%, $\left.31 \mathrm{mg}\right)$

M.p. $74-76{ }^{\circ} \mathrm{C} .{ }^{1} \mathrm{H}$ NMR $\left(\mathrm{CDCl}_{3}, 300 \mathrm{MHz}\right): \delta=7.26(\mathrm{~d}, J=8.6 \mathrm{~Hz}, 2 \mathrm{H}), 6.86(\mathrm{~d}, J=8.6 \mathrm{~Hz}, 2 \mathrm{H})$, $3.99(\mathrm{tt}, J=11.7,4.4 \mathrm{~Hz}, 1 \mathrm{H}), 3.79(\mathrm{~s}, 3 \mathrm{H}), 3.59(\mathrm{dd}, J=11.2,2.3 \mathrm{~Hz}, 1 \mathrm{H}), 3.22(\mathrm{ddd}, J=12.2,4.4$, $2.6 \mathrm{~Hz}, 1 \mathrm{H}), 2.79(\mathrm{td}, J=12.3,2.5 \mathrm{~Hz}, 1 \mathrm{H}), 2.28$ (dquint, $J=12.5,2.3 \mathrm{~Hz}, 1 \mathrm{H}), 2.19$ (dsex, $J=12.6$, $2.3 \mathrm{~Hz}, 1 \mathrm{H}), 1.91-1.74(\mathrm{~m}, 3 \mathrm{H}) .{ }^{13} \mathrm{C} \mathrm{NMR}\left(\mathrm{CDCl}_{3}, 75 \mathrm{MHz}\right): \delta=159.1(\mathrm{C}), 135.6(\mathrm{C}), 127.8(2 \times$ $\mathrm{CH}), 114.0(2 \times \mathrm{CH}), 61.1(\mathrm{CH}), 57.8(\mathrm{CH}), 55.4\left(\mathrm{CH}_{3}\right), 46.5\left(\mathrm{CH}_{2}\right), 45.5\left(\mathrm{CH}_{2}\right), 37.0\left(\mathrm{CH}_{2}\right)$. ESIHRMS calculated for $\mathrm{C}_{12} \mathrm{H}_{17} \mathrm{NOCl}[\mathrm{M}+\mathrm{H}]^{+} 226.0998$, found 226.0998 .

4-chloro-2-p-tolylpiperidine cis $7 \mathbf{f}$ White solid (70\%, $58 \mathrm{mg})$

M.p. $78-80{ }^{\circ} \mathrm{C} .{ }^{1} \mathrm{H} \mathrm{NMR}\left(\mathrm{CDCl}_{3}, 300 \mathrm{MHz}\right): \delta=7.23(\mathrm{~d}, J=8.0 \mathrm{~Hz}, 2 \mathrm{H}), 7.13(\mathrm{~d}, J=8.0 \mathrm{~Hz}, 2 \mathrm{H})$, $3.98(\mathrm{tt}, J=11.7,4.4 \mathrm{~Hz}, 1 \mathrm{H}), 3.58(\mathrm{dd}, J=11.3,2.3 \mathrm{~Hz}, 1 \mathrm{H}), 3.21(\mathrm{ddd}, J=12.2,4.3,2.6 \mathrm{~Hz}, 1 \mathrm{H})$, $2.77(\mathrm{td}, J=12.3,2.5 \mathrm{~Hz}, 1 \mathrm{H}), 2.32(\mathrm{~s}, 3 \mathrm{H}), 2.27$ (dquint, $J=12.5,2.2 \mathrm{~Hz}, 1 \mathrm{H}), 2.17(\mathrm{dsex}, J=12.5$, $2.3 \mathrm{~Hz}, 1 \mathrm{H}), 1.90-1.74(\mathrm{~m}, 2 \mathrm{H}), 1.67(\mathrm{~s}, 1 \mathrm{H}) .{ }^{13} \mathrm{C} \mathrm{NMR}\left(\mathrm{CDCl}_{3}, 75 \mathrm{MHz}\right): \delta=140.5(\mathrm{C}), 137.2(\mathrm{C})$, $129.3(2 \times \mathrm{CH}), 126.5(2 \times \mathrm{CH}), 61.3(\mathrm{CH}), 57.8(\mathrm{CH}), 46.5\left(\mathrm{CH}_{2}\right), 45.5\left(\mathrm{CH}_{2}\right), 37.0\left(\mathrm{CH}_{2}\right), 21.1$ $\left(\mathrm{CH}_{3}\right)$. ESI-HRMS calculated for $\mathrm{C}_{12} \mathrm{H}_{17} \mathrm{NCl}[\mathrm{M}+\mathrm{H}]^{+} 210.1049,210.1051$.

4-chloro-2-isopropylpiperidine cis 7g Orange oil (88\%, 57 mg)

${ }^{1} \mathrm{H} \mathrm{NMR}\left(\mathrm{CDCl}_{3}, 300 \mathrm{MHz}\right): \delta=3.90(\mathrm{tt}, J=11.7,4.3 \mathrm{~Hz}, 1 \mathrm{H}), 3.17(\mathrm{ddd}, J=12.6,4.5,2.5 \mathrm{~Hz}, 1 \mathrm{H})$, $2.64(\mathrm{td}, J=12.6,2.5 \mathrm{~Hz}, 1 \mathrm{H}), 2.30(\mathrm{ddd}, J=11.3,5.8,2.2 \mathrm{~Hz}, 1 \mathrm{H}), 2.21-2.10(\mathrm{~m}, 2 \mathrm{H}), 1.82$ (bs, 
$\mathrm{NH}), 1.70(\mathrm{qd}, J=11.9,4.5 \mathrm{~Hz}, 1 \mathrm{H}), 1.64-1.58(\mathrm{~m}, 1 \mathrm{H}), 1.42(\mathrm{q}, J=11.9 \mathrm{~Hz}, 1 \mathrm{H}), 0.94(\mathrm{~d}, J=2.6$ $\mathrm{Hz}, 3 \mathrm{H}), 0.92(\mathrm{~d}, J=2.6 \mathrm{~Hz}, 3 \mathrm{H}) .{ }^{13} \mathrm{C} \mathrm{NMR}\left(\mathrm{CDCl}_{3}, 75 \mathrm{MHz}\right): \delta=62.6(\mathrm{CH}), 58.6(\mathrm{CH}), 46.3$ $\left(\mathrm{CH}_{2}\right), 40.6\left(\mathrm{CH}_{2}\right), 37.7\left(\mathrm{CH}_{2}\right), 33.1(\mathrm{CH}), 19.0\left(\mathrm{CH}_{3}\right), 18.8\left(\mathrm{CH}_{3}\right)$. ESI-HRMS calculated for $\mathrm{C}_{8} \mathrm{H}_{17} \mathrm{NCl}[\mathrm{M}+\mathrm{H}]^{+}$162.1049, found 162.1049.

4-chloro-2-phenyl-1-tosylpiperidine trans $\mathbf{1 1}^{2 \mathrm{a}}$ Yellow solid (93\%, $130 \mathrm{mg}$ )

M.p. $104-106{ }^{\circ} \mathrm{C} .{ }^{1} \mathrm{H}$ NMR $\left(\mathrm{CDCl}_{3}, 300 \mathrm{MHz}\right): \delta=7.78(\mathrm{~d}, J=8.2 \mathrm{~Hz}, 2 \mathrm{H}), 7.35-7.26(\mathrm{~m}, 7 \mathrm{H}), 5.40$ (d, $J=5.0 \mathrm{~Hz}, 1 \mathrm{H}), 3.99-3.86(\mathrm{~m}, 2 \mathrm{H}), 3.09-2.99(\mathrm{~m}, 1 \mathrm{H}), 2.73$ (dquint, $J=13.6,1.9 \mathrm{~Hz}, 1 \mathrm{H}), 2.46$ (s, $3 \mathrm{H}), 1.94-1.83$ (m, $2 \mathrm{H}), 1.64-1.50(\mathrm{~m}, 1 \mathrm{H}) .{ }^{13} \mathrm{C} \mathrm{NMR}\left(\mathrm{CDCl}_{3}, 75 \mathrm{MHz}\right): \delta=143.7(\mathrm{C}), 138.1(\mathrm{C})$, $137.4(\mathrm{C}), 130.1(2 \times \mathrm{CH}), 129.1(2 \times \mathrm{CH}), 127.5(\mathrm{C}), 127.0(2 \times \mathrm{CH}), 126.6(2 \times \mathrm{CH}), 56.1(\mathrm{CH})$, $52.9(\mathrm{CH}), \quad 41.5\left(\mathrm{CH}_{2}\right), \quad 37.7\left(\mathrm{CH}_{2}\right), 35.3\left(\mathrm{CH}_{2}\right), 21.7\left(\mathrm{CH}_{2}\right)$. ESI-HRMS calculated for $\mathrm{C}_{18} \mathrm{H}_{20} \mathrm{NO}_{2} \mathrm{ClNaS}[\mathrm{M}+\mathrm{Na}]^{+} 372.0801$, found 372.0802 .

1-benzyl-4-chloro-2-phenylpiperidine 12 (64\%, $73 \mathrm{mg}$, dr: 52:48)

Cis 12: White solid M.p. $134-136{ }^{\circ} \mathrm{C} .{ }^{1} \mathrm{H}$ NMR $\left(\mathrm{CDCl}_{3}, 300 \mathrm{MHz}\right): \delta=7.44(\mathrm{~d}, J=7.1 \mathrm{~Hz}, 2 \mathrm{H}), 7.36-$ $7.19(\mathrm{~m}, 8 \mathrm{H}), 3.92(\mathrm{tt}, J=11.6,4.2 \mathrm{~Hz}, 1 \mathrm{H}), 3.26(\mathrm{ABq}, 2 \mathrm{H}), 3.20(\mathrm{dd}, J=11.3,2.7 \mathrm{~Hz}, 1 \mathrm{H}), 3.00$ $(\mathrm{dd}, J=11.2,3.0 \mathrm{~Hz}, 1 \mathrm{H}), 2.30-2.23(\mathrm{~m}, 1 \mathrm{H}), 2.12-1.87(\mathrm{~m}, 4 \mathrm{H}) .{ }^{13} \mathrm{C} \mathrm{NMR}\left(\mathrm{CDCl}_{3}, 75 \mathrm{MHz}\right): \delta=$ $143.5(\mathrm{C}), 139.2(\mathrm{C}), 128.9(2 \times \mathrm{CH}), 128.7(2 \times \mathrm{CH}), 128.3(2 \times \mathrm{CH}), 127.6(\mathrm{CH}), 127.5(2 \times \mathrm{CH})$, $126.9(\mathrm{CH}), 68.5(\mathrm{CH}), 58.8\left(\mathrm{CH}_{2}\right), 57.3(\mathrm{CH}), 52.1\left(\mathrm{CH}_{2}\right), 46.7\left(\mathrm{CH}_{2}\right), 36.8\left(\mathrm{CH}_{2}\right)$. ESI-HRMS calculated for $\mathrm{C}_{18} \mathrm{H}_{21} \mathrm{NCl}[\mathrm{M}+\mathrm{H}]^{+}$286.1362, found 286.1361. Trans 12: White solid M.p. 126-128 ${ }^{\circ} \mathrm{C}$. ${ }^{1} \mathrm{H} \mathrm{NMR}\left(\mathrm{CDCl}_{3}, 300 \mathrm{MHz}\right): \delta=7.47(\mathrm{~d}, J=7.1 \mathrm{~Hz}, 2 \mathrm{H}), 7.35-7.19(\mathrm{~m}, 8 \mathrm{H}), 4.51(\mathrm{t}, J=2.9 \mathrm{~Hz}, 1$ $\mathrm{H}), 3.70(\mathrm{dd}, J=8.5,5.5 \mathrm{~Hz}, 1 \mathrm{H}), 3.34(\mathrm{ABq}, 2 \mathrm{H}), 2.78(\mathrm{dt}, J=11.9,3.5 \mathrm{~Hz}, 1 \mathrm{H}), 2.54(\mathrm{td}, J=12.1$, $2.6 \mathrm{~Hz}, 1 \mathrm{H}), 2.10-2.06(\mathrm{~m}, 3 \mathrm{H}), 1.88(\mathrm{~d}, J=14.2 \mathrm{~Hz}, 1 \mathrm{H}) .{ }^{13} \mathrm{C} \mathrm{NMR}\left(\mathrm{CDCl}_{3}, 75 \mathrm{MHz}\right): \delta=144.2$ (C), $139.6(\mathrm{C}), 128.8(2 \times \mathrm{CH}), 128.7(2 \times \mathrm{CH}), 128.2(2 \times \mathrm{CH}), 127.7(2 \times \mathrm{CH}), 127.4(\mathrm{CH}), 126.8$ (CH), $62.5(\mathrm{CH}), 59.4\left(\mathrm{CH}_{2}\right), 57.8(\mathrm{CH}), 46.3\left(\mathrm{CH}_{2}\right), 43.8\left(\mathrm{CH}_{2}\right), 33.6\left(\mathrm{CH}_{2}\right)$. ESI-HRMS calculated for $\mathrm{C}_{18} \mathrm{H}_{21} \mathrm{NCl}[\mathrm{M}+\mathrm{H}]^{+}$286.1362, found 286.1361.

4-chloro-2-phenyl-1-propylpiperidine $\mathbf{1 3}$ (90\%, $85 \mathrm{mg}$, dr: 57:43)

Cis 13: Yellow oil ${ }^{1} \mathrm{H}$ NMR $\left(\mathrm{CDCl}_{3}, 300 \mathrm{MHz}\right): \delta=7.32-7.24(\mathrm{~m}, 5 \mathrm{H}), 3.92(\mathrm{tt}, J=11.7,4.3 \mathrm{~Hz}, 1$ H), $3.20(\mathrm{dt}, J=11,3,3.2 \mathrm{~Hz}, 1 \mathrm{H}), 3.08(\mathrm{dd}, J=11.3,2.5 \mathrm{~Hz}, 1 \mathrm{H}), 2.37-2.30(\mathrm{~m}, 1 \mathrm{H}), 2.24-2.16(\mathrm{~m}$, 
$2 \mathrm{H}), 2.10-1.79(\mathrm{~m}, 4 \mathrm{H}), 1.36(\mathrm{q}, J=7.6 \mathrm{~Hz}, 2 \mathrm{H}), 0.71(\mathrm{t}, J=7.3 \mathrm{~Hz}, 3 \mathrm{H}) .{ }^{13} \mathrm{C} \mathrm{NMR}\left(\mathrm{CDCl}_{3}, 75\right.$ MHz): $\delta=143.6(\mathrm{C}), 128.7(2 \times \mathrm{CH}), 127.6(2 \times \mathrm{CH}), 127.4(\mathrm{CH}), 68.5(\mathrm{CH}), 57.4(\mathrm{CH}), 56.2\left(\mathrm{CH}_{2}\right)$, 52.0 $\left(\mathrm{CH}_{2}\right), 46.6\left(\mathrm{CH}_{2}\right), 37.0\left(\mathrm{CH}_{2}\right), 19.5\left(\mathrm{CH}_{2}\right), 11.8\left(\mathrm{CH}_{3}\right)$. ESI-HRMS calculated for $\mathrm{C}_{14} \mathrm{H}_{21} \mathrm{NCl}$ $[\mathrm{M}+\mathrm{H}]^{+}$238.1362, found 238.1359. Trans 13: Yellow oil ${ }^{1} \mathrm{H}$ NMR $\left(\mathrm{CDCl}_{3}, 300 \mathrm{MHz}\right): \delta=7.35-7.23$ $(\mathrm{m}, 5 \mathrm{H}), 4.51$ (quint, $J=2.9 \mathrm{~Hz}, 1 \mathrm{H}), 3.59(\mathrm{dd}, J=8.9,4.7 \mathrm{~Hz}, 1 \mathrm{H}), 2.97(\mathrm{dt}, J=11.9,3.2 \mathrm{~Hz}, 1 \mathrm{H})$, $2.64(\mathrm{td}, J=12.0,2.1 \mathrm{~Hz}, 1 \mathrm{H}), 2.44-2.34(\mathrm{~m}, 1 \mathrm{H}), 2.18(\mathrm{t}, J=13.1 \mathrm{~Hz} 1 \mathrm{H}), 2.01-1.97(\mathrm{~m}, 4 \mathrm{H})$, 1.44-1.37 (m, $2 \mathrm{H}), 0.72(\mathrm{t}, J=7.4 \mathrm{~Hz}, 3 \mathrm{H}) .{ }^{13} \mathrm{C} \mathrm{NMR}\left(\mathrm{CDCl}_{3}, 75 \mathrm{MHz}\right): \delta=144.1(\mathrm{C}), 128.6(2 \times$ $\mathrm{CH}), 127.8(2 \times \mathrm{CH}), 127.2(\mathrm{CH}), 62.2(\mathrm{CH}), 57.9(\mathrm{CH}), 56.8\left(\mathrm{CH}_{2}\right), 46.3\left(\mathrm{CH}_{2}\right), 43.6\left(\mathrm{CH}_{2}\right), 33.7$ $\left(\mathrm{CH}_{2}\right), 19.4\left(\mathrm{CH}_{2}\right), 11.9\left(\mathrm{CH}_{3}\right)$. ESI-HRMS calculated for $\mathrm{C}_{14} \mathrm{H}_{21} \mathrm{NCl}[\mathrm{M}+\mathrm{H}]^{+}$238.1362, found 238.1362.

\section{Supporting Information}

Copies of 1H NMR and 13C NMR spectra for products 1-4-8-9-10, for all piperidines 3-5-7-11-12-13 and X-ray crystallographic data, and crystal structure of compounds trans $\mathbf{3 e}$ and cis $\mathbf{7 a}$ are provided. The Supporting Information is available free of charge on the ACS Publications website at DOI:

\section{Author information}

Corresponding author

*E-mail: pierre.van-de-weghe@univ-rennes1.fr

*E-mail: claudia.lalli@univ-rennes1.fr

The authors declare no competing financial interest.

\section{Acknowledgments}

We thank Université de Rennes 1, CNRS, Rennes Métropole and Région Bretagne for financial support. The CRMPO de l'Institut des Sciences Chimiques de Rennes is gratefully acknowledged for mass measurement.

\section{References}

1. (a) Pastor, I. M.; Yus, M. Curr. Org. Chem. 2012, 16, 1277. (b) Olier, C.; Kaafarini, M.; Gastaldi, S.; Bertrand, M. P. Tetrahedron 2010, 66, 413. 
2. (a) Carballo, R. M.; Ramírez, M. A.; Rodríguez, M. L.; Martín, V. S.; Padrón, J. I. Org. Lett. 2006, 8, 3837. (b) Miranda, P. O.; Carballo, R. M.; Martín, V. S.; Padrón, J. I. Org. Lett. 2009, 11, 357. (c) Carballo, R. M.; Valdomir, G.; Purino, M.; Martín, V. S.; Padrón, J. I. Eur. J. Org. Chem. 2010, 12, 2304. (d) Pérez, S. J.; Purino, M.; Miranda, P. O.; Martín, V. S.; Fernández, I.; Padrón, J. I. Chem. Eur. J. 2015, 21, 15211.

3. Murty, M. S. R.; Ram, K. R.; Yadav, J. S. Tetrahedron Lett. 2008, 49, 1141.

4. (a)Yadav, J. S.; Subba Reddy, B. V.; Ramesh, K.; Narayana Kumar, G. G. K. S.; Grée, R. Tetrahedron Lett. 2010, 51, 818. (b) Launay, G. G.; Slawin, A. M. Z.; O’Hagan, D. Beilstein J. Org. Chem. 2010, 6, No. 41.

5. Dobbs, A. P.; Guesné, S. J. J.; Parker, R. J.; Skidmore, J.; Stephenson, R. A.; Hursthouse, M. B. Org. Biomol. Chem. 2010, 8, 1064.

6. (a) Sabitha, G.; Das, S. K.; Srinivas, R.; Yadav, J. S. Helv. Chim. Acta 2010, 93, 2023. (b) Clarisse, D.; Pelotier, B.; Fache, F. Chem.-Eur. J. 2013, 19, 857.

7. Yadav, J. S.; Subba Reddy, B. V.; Chaya, D. N.; Narayana Kumar, G. G. K. S.; Naresh, P.; Jagadeesh, B. Tetrahedron Lett. 2009, 50, 1799.

8. Yadav, J. S.; Subba Reddy, B. V.; Ramesh, K.; Narayana Kumar, G. G. K. S.; Grée, R. Tetrahedron Lett. 2010, 51, 1578.

9. Subba Reddy, B. V.; Ramesh, K.; Ganesh, A. V.; Narayana Kumar, G. G. S. K.; Yadav, J. S.; Grée, R. Tetrahedron Lett. 2011, 52, 495.

10. (a) Frank, K. E.; Aubé, J. Tetrahedron Lett. 1998, 39, 7239. (b) Frank, K. E.; Aubé, J. J. Org. Chem. 2000, 65, 655. (c) Dobbs, A. P.; Guesné, S. J. J.; Hursthouse, M. B.; Coles, S. J. Synlett 2003, 1740. (d) Dobbs, A. P.; Guesné, S. J. J.; Martinović, S.; Coles, S. J.; Hursthouse, M. B. J. Org. Chem. 2003, 68, 7880. (e) Parchinsky, V.; Shumsky, A.; Krasavin, M. Tetrahedron Lett. 2011, 52, 7157. (f) Liu, X.; McCormack, M. P.; Waters, S. P. Org. Lett. 2012, 14, 5574. (g) Colin, O.; Greck, C.; Prim, D.; Thomassigny, C. Eur. J. Org. Chem. 2014, 7000. (h) Chio, F. K. I.; Guesné, S. J. J.; Hassall, L.; McGuire, T.; Dobbs, A. P. J. Org. Chem. 2015, 80, 9868. 
11. (a) Jacolot, M.; Jean, M.; Levoin, N.; van de Weghe, P. Org. Lett. 2012, 14, 58. (b) Bokar, P.; van de Weghe, P.; Subba Reddy, B. V.; Yadav, J. S.; Grée, R. Chem. Commun. 2012, 48, 9316. (c) Lalli, C.; van de Weghe, P. Chem. Commun. 2014, 50, 7495.

12. In order to ascertain whether the reaction is under kinetic of thermodynamic control, cis- and trans-3a were separately subjected to the reaction conditions $\left(\mathrm{TiCl}_{4}(1 \mathrm{eq}), p-\mathrm{TsOH} . \mathrm{H}_{2} \mathrm{O}(10 \mathrm{~mol} \%)\right.$, $\mathrm{CH}_{2} \mathrm{Cl}_{2}, 60{ }^{\circ} \mathrm{C}, 16 \mathrm{~h}$ ). Cis-trans interconversion was not observed, indicating that the reaction is irreversible and proceeds under kinetic control.

13. Kishi, Y.; Inagi, S.; Fuchigami, T. Eur. J. Org. Chem., 2009, 103.

14. See supporting information.

15. Lukowski, M.; Jacobs, K.; Hsueh, P.; Lindsay, H. A.; Milletti, M. C. Tetrahedron 2009, 65, 10311.

16. Mizutani, T.; Ukaji, Y.; Inomata, K. Bull. Chem. Soc. Jpn. 2003, 76, 1251.

17. Taillier, C.; Hameury, T.; Bellosta, V.; Cossy, J. Tetrahedron 2007, 63, 4472. 